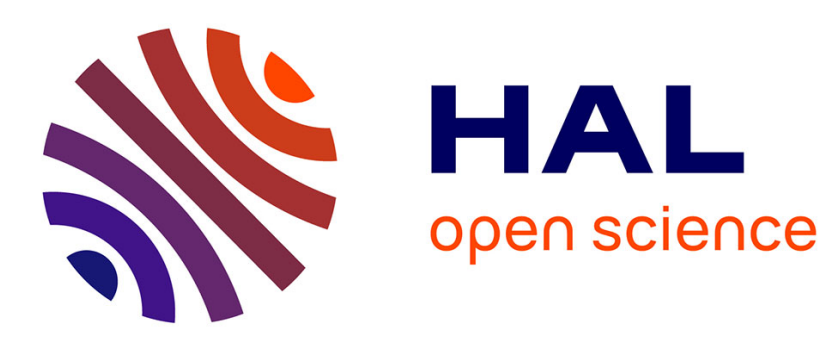

\title{
Traffic-Aware Scheduling and Feedback Allocation in Multichannel Wireless Networks
}

Matha Deghel, Mohamad Assaad, Mérouane Debbah, Anthony Ephremides

\section{To cite this version:}

Matha Deghel, Mohamad Assaad, Mérouane Debbah, Anthony Ephremides. Traffic-Aware Scheduling and Feedback Allocation in Multichannel Wireless Networks. IEEE Transactions on Wireless Communications, 2018, 17 (8), pp.5520-5534. 10.1109/twc.2018.2845406 . hal-01804844

\section{HAL Id: hal-01804844 \\ https://hal.science/hal-01804844}

Submitted on 18 Jun 2020

HAL is a multi-disciplinary open access archive for the deposit and dissemination of scientific research documents, whether they are published or not. The documents may come from teaching and research institutions in France or abroad, or from public or private research centers.
L'archive ouverte pluridisciplinaire HAL, est destinée au dépôt et à la diffusion de documents scientifiques de niveau recherche, publiés ou non, émanant des établissements d'enseignement et de recherche français ou étrangers, des laboratoires publics ou privés. 


\title{
Traffic-Aware Scheduling and Feedback Allocation in Multichannel Wireless Networks
}

\author{
Matha Deghel, Student, IEEE, Mohamad Assaad, Senior, IEEE, \\ Mérouane Debbah, Fellow, IEEE, and Anthony Ephremides, Life Fellow, IEEE
}

\begin{abstract}
This work studies the problem of feedback allocation and scheduling for a multichannel downlink cellular network under limited and delayed feedback. We propose two efficient algorithms that select the link states that should be reported to the base-station (BS). A novelty here is that these feedback allocation algorithms are performed at the users' side to take advantage of their local CSI (channel state information) knowledge in order to achieve higher gains. The first algorithm is suitable for a continuous-time contention scheme and requires only one feedback per channel, whereas the second one is adapted for a discrete-time contention scheme and adopts a threshold-based concept. For this second algorithm, we study some implementation aspects related to the feedback period and investigate the trade-off between knowing at the BS a small number of accurate link states and a larger but outdated number of link states. We show that these algorithms, combined with the Max-Weight scheduling, achieve good stability performance compared with the ideal system.
\end{abstract}

\section{INTRODUCTION}

In this work, we address the problem of joint feedback reporting and scheduling for multiuser downlink wireless networks employing multiple parallel channels, i.e. multicarrier technique, to serve the users. Such a setting corresponds for example to a single cell orthogonal frequency-division multiplexing access (OFDMA) scheme, which is implemented in the long term evolution (LTE) standards [2] and was shown to deliver a substantial increase in the system's performance; OFDMA is also the multiple access technique adopted for $5 \mathrm{G}$ systems. To exploit multiuser diversity in multichannel downlink networks, the base station (BS) needs to acquire channel state information (CSI) from users. These CSIs are usually unknown at the BS, especially in frequency-division duplex (FDD) systems which lack of channel reciprocity. A common method to acquire the downlink CSI is to allocate a

M. Deghel and M. Assaad are with the TCL Chair on 5G, Laboratoire de Signaux et Systèmes (L2S, UMR8506), CentraleSupélec, 91192 Gif-sur-Yvette, France (e-mail: matha.deghel@centralesupelec.fr; mohamad.assaad@centralesupelec.fr).

M. Debbah is with the Large Systems and Networks Group (LANEAS), CentraleSupélec, Gif-sur-Yvette, France (merouane.debbah@centralesupelec.fr), and also with the Mathematical and Algorithmic Sciences Lab, Huawei Technologies Co. Ltd., France (e-mail: merouane.debbah@huawei.com).

A. Ephremides is with the Department of Electrical and Computer Engineering and Institute for Systems Research University of Maryland, College Park, MD 20742 (e-mail: etony@umd.edu).

Parts of this paper have been presented at the IEEE Global Communications Conference (GLOBECOM), Washington DC, USA, 2016 [1].

This research has been performed in the framework of the Horizon 2020 project ONE5G (ICT-760809) receiving funds from the European Union. part of the uplink resources to the users to report their CSIs. However, the more CSIs are decided to be reported, the more resources are needed, thus resulting in a bigger overhead in the system. In this regard, in [3]-[8] different approaches are proposed to reduce the feedback load while still achieving the benefits of multiuser diversity. However, these works do not take into account the incoming traffic processes of the users. In this work, an important factor that is considered is the traffic pattern for each user, which is stored in a respective queue at the BS. Hence, the system stability (i.e. all the queue lengths are finite) is an important property the scheduling mechanism should take into account [9], [10].

The feedback allocation algorithm directly impacts the scheduling mechanism and thus the system stability since a user cannot be scheduled unless its CSI is reported to the scheduler. In realistic scenarios, complete feedback knowledge is not readily available at the scheduler. Different limitations result in such incomplete information, such as estimation error, delay and limited feedback resources, so it is important to analyze the impact of such imperfections on the system stability. For a single channel system, mainly time-division duplex (TDD), the authors in [11], [12] have proposed a feedback allocation scheme. Specifically, this scheme sets as threshold the rate of the user with the maximum queue length and requires no knowledge of channel and traffic statistic. In addition, distributed scheduling approach is considered in [13] where the authors propose a Greedy Maximal Scheduling, for which the achievable stability performance depends on the network topology. Some other distributed schemes that approach the system stability region are provided in [14], [15], but are hard to implement. Moreover, the work in [16] derives the optimal feedback scheme for a single-channel downlink system under partial channel state information. In [17], the authors study centralized scheduling with rate adaptation under imperfect channel-estimator joint statistics. Under a multichannel downlink system where an FDD mode is adopted, obtaining complete feedback would require a prohibitive portion of the overall uplink capacity, especially for large number of users. Under such a system, the authors in [18] study the impact of limited feedback resources on the achievable stability region. However, in this latter work the delay in the feedback process is not accounted for. Also in the context of multichannel wireless networks, the authors in [19] propose a set of low computational complexity scheduling algorithms with a large number of users and proportionally large bandwidth. Furthermore, in [20] the problem of routing/scheduling in a wireless network with delayed network (channel and queue) 
state information (NSI) is studied. Specifically, two cases are considered: the centralized routing/scheduling, where a central controller obtains heterogeneously delayed information from each of the nodes, and the decentralized routing/scheduling, where each node makes a decision based on its current channel and queue states along with homogeneous delayed NSI from other nodes. On the other hand, the authors in [21] analyze the effect that delayed CSI has on the throughput performance of scheduling for a single channel wireless network.

In this paper, we consider a multichannel multiuser wireless downlink network where both limited feedback resources and delayed feedback information are accounted for. To the best of our knowledge, this is the first work to account for these two imperfections at the same time for a multichannel system where the incoming traffic processes of the users are taken into consideration. The incoming data for each user is stored in a respective buffer at the BS. Let $L$ be the total number of channels in the system. Note that the term "channel" denotes a certain frequency band, whereas the term "link" refers to the wireless connection between a user and the BS over a specific frequency band. Regarding the feedback resources, we adopt a setting where the feedback capacity per slot is limited; in practice (e.g. LTE systems), at a given slot it is not possible to feed back the CSI for all the users on all the channels. Specifically, only $\bar{F}$ link states (i.e. CSI) can be reported to the BS per slot and the link states used in the scheduling are delayed. Since the feedback process directly impacts the scheduling mechanism, we design efficient joint feedback reporting and scheduling algorithms. Note that such algorithms can be designed using Partially Observable Markov Decision Process (POMDP) formulation, in which the feedback decision at each time depends on the previous feedback decisions. Solutions for MDP or POMDP problems are known to be complex and most-likely not suitable for implementation in practice. In order to avoid such complexity, we have designed simple memoryless algorithms (i.e. the decision at time $t$ does not take into account the previous feedback decisions) based on Max-Weight policy. Note that the performance of these algorithms are measured w.r.t. the performance of the ideal system (where all the link states are fully and perfectly known at the BS at no cost).

The main contributions of the paper are summarized as follows:

- We first propose an algorithm that uses exactly $L$ feedback resources, where the feedback and scheduling decisions are done at the users' side; such an approach is considered to take advantage of the local CSI knowledge of these users in order to achieve better gains. This algorithm has the advantage to require only one feedback per channel (limited feedback), but it works under the assumption of continuous time for contention. We investigate analytically the stability performance of this algorithm and show that it can guarantee a certain fraction from the stability region of the ideal system.

- Under a discrete-time contention scheme, we propose a second (heuristic) algorithm that imitates the first algorithm as much as possible. The proposed algorithm here uses a threshold-based concept and consists in having $F$
$(>L)$ feedback resources and in letting the users decide whether they should send their CSIs or not, and then the BS performs scheduling over each channel. An efficient approach to update the threshold value is also provided. For this algorithm, the decision is memoryless and the system can decide to report an amount of feedback $F>\bar{F}$, thus the feedback process will require more than one slot in order to be accomplished. Hence, the amount of feedback resources and the delay in the feedback process are coupled. We investigate numerically under various system setups the period duration of the feedback decision in order to find a trade-off between having more $F$, which leads to a greater number of reported link states but which are more outdated, and having a lower number of reported link states but which are less outdated.

It is important to highlight that our work differs from existing works (some of which were cited earlier) that propose feedback and scheduling algorithms in the sense that (i) we account for the traffic patterns, meaning that our proposed feedback and scheduling algorithms account for the queue length of each user and do not consider a full buffer scenario, and (ii) limited and delayed feedback is considered. It is also important to note that the use of an OFDMA-like access technique is motivated by the fact that such a technique is used in LTE systems and will be also used in 5G systems. However, the scheduling algorithms adopted in LTE, such as PF (Proportional Fair), are not optimal in terms of accounting for the traffic patterns and for the limitation and imperfection in the CSI information. The same remark can be made for the feedback approaches and for the amount of feedback resources that are considered under such systems. Furthermore, since the feedback impacts the scheduling mechanism, it is essential to jointly design the feedback and scheduling algorithms in such a way as to achieve the best possible stability performance.

The rest of this paper is organized as follows. In Section II we present the system model, the feedback scheme, and the queueing model. In Section III we present a stability analysis for the adopted system. Specifically, we propose two joint feedback and scheduling algorithms, and we analyze their queueing stability performance. Numerical results and relevant discussions are carried out in Section IV. We finally conclude the paper in Section V.

\section{SySTEM MODEL}

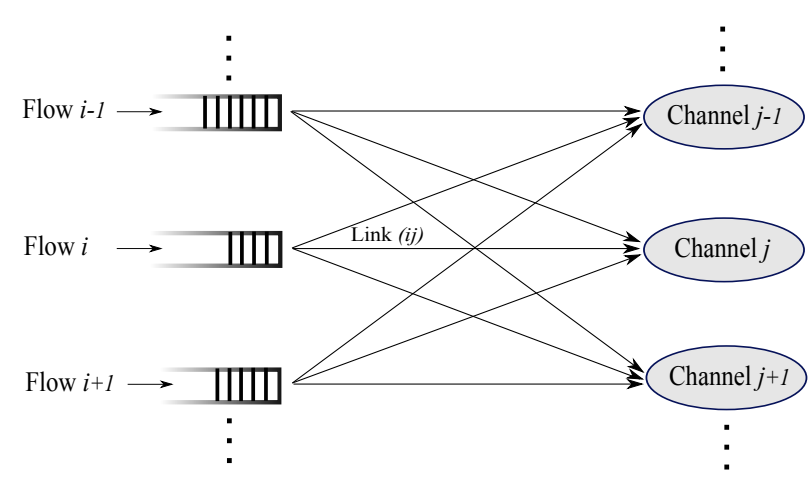

Figure 1: Multi-channel multi-user queueing system. 
We consider an FDD cellular wireless network, with one single-antenna BS, $N$ single-antenna mobile users and $L$ channels; e.g. LTE-OFDMA system. Data packets to be transmitted to the users are stored in $N$ separate queues at the BS. Time is slotted, with the slots of all users synchronized. Let $q_{i}(t)$ denote the length of queue $i$ at the beginning of time-slot $t$, and let $\mathbf{q}(t)=\left(q_{1}(t), \ldots, q_{N}(t)\right)$. This system can be seen as a multi-queue, multi-server, discrete-time queueing system. It can be noticed that since we work under an OFDMA-like system, at a given slot a channel can be allocated to one and at most one user.

We denote by $h_{i j}(t)$ the fading of the link connecting the $\mathrm{BS}$ and user $i$ using channel $j$. The received signal for the $i$ th user if it gets scheduled on the $j$ th channel at time-slot $t$ is given as

$$
\sqrt{P} h_{i j}(t) x_{i j}(t)+z_{i j}(t),
$$

where $x_{i j}(t)$ is the corresponding complex-valued data stream, with $\mathbb{E}\left\{\left|x_{i j}\right|^{2}\right\}=1, P$ is the transmission power on each channel, and $z_{i j}(t)$ is the additive white Gaussian noise with zero mean and variance $\sigma_{\mathrm{z}, i j}^{2}$, i.e. $z_{i j}(t) \sim \mathcal{C N}\left(0, \sigma_{\mathrm{z}, i j}^{2}\right)$. We assume that at slot $t$ user $i$ has perfect knowledge of $h_{i j}(t), \forall j$; the BS sends training sequences in the downlink so that each user can estimate its $h_{i j}(t)$. The corresponding signal-to-noise ratio $(\mathrm{SNR})$ is denoted and given as

$$
\gamma_{i j}(t)=\rho_{i j}\left|h_{i j}(t)\right|^{2}, \text { with } \rho_{i j}=\frac{P}{\sigma_{\mathrm{z}, i j}^{2}} .
$$

In any time-slot, the link state of a user on a channel represents the number of packets that can be successfully transmitted without outage to that user, on that channel. Transmission at a rate above the link state always fails, whereas transmission at a rate below or equal to the link state always succeeds. We use $C_{i j}(t)$ to represent the state of link $(i j)$ at time-slot $t$. We assume that each link state can take $K$ possible values $\left\{R_{1}, R_{2}, \ldots, R_{K}\right\}$, where rate $R_{k}$ represents state $k$ and corresponds to the case where $\tau_{k} \leq \gamma_{i j}(t)<\tau_{k+1}$, for some non-negative thresholds $\tau_{k}$; it can be easily noticed that $R_{k}>R_{m}$ for $k>m$. This setting is very used in practice where usually $K$ modulation and coding schemes are used (i.e. the instantaneous transmitted rate can take only $K$ possible values).

Finally, the fading process, which is represented by the coefficients $h_{i j}(t)$, is assumed to be channel convergent [22]. For channel convergent processes, it is known that the time average fraction of time in each state converges to the steady state distribution, and the expected time average is arbitrarily close to this distribution if sampled over a suitably large interval (of time); in the special case of i.i.d. (independently and identically distributed) fading process, the steady state averages are achieved every time-slot. Note that this general model is widely used to model the fact that each fading coefficient is Markovian and that it is not necessarily i.i.d. over slots.

\section{A. Limited and Delayed CSI Knowledge Scheme}

Before presenting the limited and delayed CSI scheme, we first provide the conditions under which the system is called ideal (in terms of CSI knowledge):
Ideal system: The system is called ideal when at each timeslot the BS has perfect and full knowledge of all the link states at no cost. In other words, at time-slot $t$ the BS knows (perfectly) $C_{i j}(t), \forall i, j$. Note that the term 'perfect' means that each $C_{i j}(t)$ is known perfectly, whereas the term 'full' refers to the fact that all these link states are known. We recall that at time-slot $t$ user $i$ knows perfectly $h_{i j}(t), \forall j$, which implies that this user has perfect knowledge of $C_{i j}(t), \forall j$. Hence, for the system to be called ideal we should assume that: (a) all the users send their perfect knowledge of their link states to the BS, (b) if the users send these states at time-slot $t$, they arrive to the BS also at time-slot $t$, and (c) there is no cost for this CSI acquisition process.

We now present the limited and delayed CSI scheme. We consider a realistic context where the feedback capacity per slot is limited. Specifically, we assume that at most $\bar{F}$ link rates can be reported per slot. Let $d$ denote the delay due to the feedback process. The users estimate the CSIs at time $t-d$ and reports a limited number of CSIs to the BS, which uses these reported CSIs to schedule the users and to transmit packets to the scheduled users at time $t$. We therefore consider a given delay $d$ between the CSI estimation and feedback decision time and the scheduling and transmission time. This assumption allows us to provide a theoretical performance analysis of the proposed schemes in the rest of this paper. In general, there is also a coupling between $d$ and the amount of feedback. This issue will be discussed in Subsection III.B. We use $\hat{h}_{i j}(t)$ to denote $h_{i j}(t-d)$, which is the fading of link $(i j)$ at timeslot $t-d$. We recall that in each slot each user has a perfect estimation of the $h_{i j}, \forall j$, of this slot. For example, at timeslot $t-d$ user $i$ knows perfectly $\hat{h}_{i j}(t)$ (i.e. $\left.h_{i j}(t-d)\right), \forall j$. The SNR corresponding to $\hat{h}_{i j}(t)$ is denoted and given by $\hat{\gamma}_{i j}(t)=\rho_{i j}\left|\hat{h}_{i j}(t)\right|^{2}$.

We use $\hat{C}_{i j}(t)$ to denote the state of link $(i j)$ at time-slot $t-d$, i.e. $\hat{C}_{i j}(t)=C_{i j}(t-d)$. Hence, $\hat{C}_{i j}(t)$ depends on the value of $\hat{\gamma}_{i j}(t)$, e.g. $\hat{C}_{i j}(t)=R_{k}$ if $\tau_{k} \leq \hat{\gamma}_{i j}(t)<\tau_{k+1}$.

\section{B. Queueing Model}

We here present the queueing model (i.e. queue dynamics) for the ideal system as well as for the system with limited and delayed feedback. To this end, we consider that each of the $N$ users has an incoming traffic process $A_{i}(t)$, which is an integer-valued process, measured in bits, i.i.d. in time and independent across users, with $A_{i}(t)<A_{\max }$, for some finite constant $A_{\max }$. The mean rate of this process is $a_{i}=\mathbb{E}\left\{A_{i}(t)\right\}$. We assume that packets arrive at the $\mathrm{BS}$ at the beginning of the time-slot and are served at the end of the time-slot.

We also assume that a transmission over link $(i j)$ can only be fulfilled if the corresponding link state (i.e. $C_{i j}(t)$ in the ideal case for example) is reported to the BS.

1) Queиe Dynamics under the Ideal System: To begin with, we define $S_{i j}(t)$ to be the scheduling decision associated with link $(i j)$ at time-slot $t$. So, we have

$$
S_{i j}(t)= \begin{cases}1, & \text { if user } i \text { gets scheduled on channel } j \\ 0, & \text { otherwise }\end{cases}
$$


As mentioned earlier, in one time-slot and on a given channel one and at most one user can be scheduled. As a result, for all $t$ and all $j$, any valid service policy must obey $\sum_{i=1}^{N} S_{i j}(t) \leq 1$. It should be pointed out that under the ideal system there is no need to define a feedback decision since all the link states are reported (without delay and at no cost).

Based on the above, the queues in the system evolve according to the following equation

$$
\begin{aligned}
& q_{i}(t+1)= \\
& \max \left\{q_{i}(t)+A_{i}(t)-\sum_{j=1}^{L} C_{i j}(t) S_{i j}(t), 0\right\}, \text { for } 1 \leq i \leq N,
\end{aligned}
$$

where $\sum_{j=1}^{L} C_{i j}(t) S_{i j}(t)$ represents the service rate allocated for user $i$ at time-slot $t$.

2) Queue Dynamics under the System with Delayed and Limited CSI: Let us define $S_{i j}(t)$ to be the scheduling decision associated with link $(i j)$ at time-slot $t$. In addition, we define $Y_{i j}(t)$ as the feedback decision associated with link $(i j)$ at time-slot $t$. Let $\hat{Y}_{i j}(t)=Y_{i j}(t-d)$ denote the feedback decision associated with link $(i j)$ at time-slot $t-d$. So, we can write

$$
\hat{Y}_{i j}(t)= \begin{cases}1, & \text { if } \hat{C}_{i j}(t) \text { gets reported to the scheduler } \\ 0, & \text { otherwise }\end{cases}
$$

where we recall that $\hat{C}_{i j}(t)=C_{i j}(t-d)$. On the other side, for $S_{i j}(t)$, which represents the scheduling decision at time-slot $t$, we have

$$
S_{i j}(t)= \begin{cases}1, & \text { if user } i \text { gets scheduled on channel } j \\ 0, & \text { otherwise }\end{cases}
$$

The queueing dynamics are then given as follows

$$
\begin{aligned}
& q_{i}(t+1)= \\
& \max \left\{q_{i}(t)+A_{i}(t)-\sum_{j=1}^{L} \hat{C}_{i j}(t) \hat{Y}_{i j}(t) S_{i j}(t) \mathbb{1}_{\left(C_{i j}(t) \geq \hat{C}_{i j}(t)\right)}, 0\right\},
\end{aligned}
$$$$
\text { for } 1 \leq i \leq N \text {, }
$$

where $\mathbb{1}_{(\cdot)}$ is the indicator function and the expression

$$
\sum_{j=1}^{L} \hat{C}_{i j}(t) \hat{Y}_{i j}(t) S_{i j}(t) \mathbb{1}_{\left(C_{i j}(t) \geq \hat{C}_{i j}(t)\right)}
$$

is the service rate allocated for user $i$ at time-slot $t$. The use of indicator function $\mathbb{1}_{\left(C_{i j}(t) \geq \hat{C}_{i j}(t)\right)}$ is due to a possible mismatch between the reported rate, $\hat{C}_{i j}(t)$, and the actual link state, $C_{i j}(t)$, i.e. it is possible that the reported rate is greater than the actual link rate, thus leading to outage.

Finally, since we are interested in evaluating the stability performance of the system, we next recall the definitions of "strong stability" of a queueing system, "stability region" of a scheduling rule, and "Max-Weight" scheduling policy.
Definition 1 (Strong Stability). The condition for strong stability can be expressed as

$$
\limsup _{T \rightarrow \infty} \frac{1}{T} \sum_{t=0}^{T-1} \mathbb{E}\left\{q_{i}(t)\right\}<\infty, \forall i \in\{1, \ldots, N\} .
$$

In the remainder of the paper "stable" will imply "strongly stable" unless stated otherwise.

Definition 2 (Stability Region). The stability region achieved by a scheduling policy is defined as the set of vectors of mean arrival rates for which the system stays stable under this policy.

Definition 3 (Max-Weight Policy). In every time-slot, the Max-Weight rule schedules the user that yields the largest product of queue length and link rate.

\section{Proposed Algorithms AND StABILITY ANALYSIS}

In this section, we provide a stability analysis for the system under consideration. Specifically, we propose a joint feedback allocation and scheduling algorithm, named FSA, under which the allocations are done at the users' side. We derive the fraction that this algorithm reaches of the stability region of the ideal system (with perfect and full feedback knowledge). Although it provides good stability performance, this algorithm is suitable for systems where continuous-time contention is used. For systems where discrete-time contention is only feasible, we propose a second algorithm, termed as FMA, that adopts a different approach and that tries to imitate FSA as much as possible. For FMA, an amount of $F$ feedback resources is available, i.e. at most $F$ links can report their CSI to the BS. We discuss the implementation of this second algorithm and investigate its best feedback time period.

In order for the BS to perform scheduling, it needs to receive the rates of the links that were selected for the feedback. The feedback allocation process is thus of great importance since it directly impacts the scheduling mechanism. Hence, a necessary step to conduct the stability analysis is to specify/develop a joint feedback allocation and scheduling algorithm and to characterize its performance w.r.t. the ideal system where full and perfect CSI is available at no cost. Obviously, under the ideal system there is no need for a feedback allocation algorithm since it is supposed that all the link states are instantaneously reported to the BS (at not cost).

We next provide the motivation behind the reasoning regarding the feedback decision used in algorithms FSA and FMA (which will be presented afterwards).

Motivation: We first note that a Max-Weight-like rule is adopted for the scheduling mechanism, under which the decisions are affected by the reported CSIs as well as the queue lengths; i.e. the feedback allocation algorithm directly impacts the scheduling mechanism. One could consider that the BS must decide of the feedback allocation. However, letting the users make this decision provides a gain due the following: if the BS decides which user should feed back its CSI, this decision will be based on the channel statistics. In other words, the BS can ask a user with bad CSI to report its feedback, since the BS cannot know beforehand if the current CSI is good or 
bad. However, the users estimate their CSI at each time-slot and therefore have perfect knowledge of their current CSIs, so including them in the feedback process would enhance the system performance. On the other hand, the users lack the queue lengths information, which should be taken into consideration by the feedback allocation since the scheduling mechanism is directly impacted by the feedback decisions. In general, the stability of the system is more sensitive to the channels variation than to the queues variation [23], so we can provide the users with the queue lengths knowledge every period of time and not every slot. This is done by the BS which broadcasts the queue lengths information every $T_{\mathrm{b}}$ slots, where $T_{\mathrm{b}}$ is typically high so that the (signaling) cost of such a broadcast stays negligible.

\section{A. Algorithm FSA and its Stability Performance}

Let 'mdl' represent the system where for each channel only one user reports its feedback and where this user is selected using algorithm FSA. For this system, we assume that the contention can be done in continuous time. We use 'pf' to denote the ideal system, where Max-Weight policy is used to schedule users for transmission. Let $\mathcal{M}^{\mathrm{pf}}(t)$ and $\mathcal{M}^{\mathrm{mdl}}(t)$ be the subsets of scheduled links at time-slot $t$ under 'pf' and 'mdl', respectively. Note that here we consider that $L$ link rates can be reported to the BS with a delay $d$. Before presenting algorithm FSA, and to better understand the reasoning that is used in this algorithm, we next provide the Max-Weight scheduling used under the ideal system. First, recall that under this system the BS has full and perfect knowledge (at no cost) of all the link states. That is to say, at time-slot $t$ the BS knows $C_{i j}(t), \forall i, j$. Thus, at time-slot $t$ the Max-Weight policy schedules over channel $j$ the user that results from the following operation

$$
\arg \max _{i}\left\{q_{i}(t) C_{i j}(t)\right\} .
$$

For this ideal system, we define $g_{\mathrm{pf}}$ to be the expected weighted throughput, given as follows

$$
g_{\mathrm{pf}}(\mathbf{q}(t))=\mathbb{E}\left\{\sum_{j=1}^{L} \sum_{i=1}^{N} q_{i}(t) C_{i j}(t) S_{i j}(t) \mid \mathbf{q}(t)\right\},
$$

where here $S_{i j}(t)$ represents the scheduling decision of link $(i j)$ at time-slot $t$ under the ideal system, which must obey $\sum_{i=1}^{N} S_{i j}(t) \leq 1$. An alternative way to write $g_{\mathrm{pf}}$ is

$$
g_{\mathrm{pf}}(\mathbf{q}(t))=\mathbb{E}\left\{\sum_{(i j) \in \mathcal{M}^{\mathrm{pf}}(t)} q_{i}(t) C_{i j}(t) \mid \mathbf{q}(t)\right\} .
$$

Algorithm FSA: For system 'mdl', the feedback and scheduling decisions are based on the following procedure.

1) Queue lengths broadcast every $T_{\mathrm{b}}$ slots:

Every $T_{\mathrm{b}}$ time-slots, that is to say, at time $0, T_{\mathrm{b}}, 2 T_{\mathrm{b}}, \ldots, n T_{\mathrm{b}}, \ldots$, the BS broadcasts the queue lengths of all users, where $T_{\mathrm{b}}$ is typically high. So each user has outdated knowledge of the state of its queue (and all other queues). Let $\tilde{q}_{i}(t)$ be the (outdated) queue length the users know at time $t$, i.e. $\tilde{q}_{i}(t)=q_{i}\left(n T_{\mathrm{b}}\right)$ for $t \in\left[n T_{\mathrm{b}},(n+1) T_{\mathrm{b}}[\right.$.
2) Feedback and scheduling decisions at time-slot $t-d$ : To simplify the notation, we denote $\tilde{q}_{i}(t-d)$ as $\hat{\tilde{q}}_{i}(t)$. For each channel $j \in\{1, \ldots, L\}$, only one user sends its CSI to the BS.

To detect this user, we use an approach that consists in letting the users contend among each other for a certain period of time. Let $T_{\mathrm{c}}$ be the contention period for each channel. Assuming that contention can be done in continuous time [24], for each channel $j \in\{1, \ldots, L\}$ the contention is performed as the following:

User $i, \forall i \in\{1, \ldots, N\}$, waits until time

$$
T_{\mathrm{c}}\left(\hat{\tilde{q}}_{i}(t) \hat{C}_{i j}(t) \mathbb{P}\left\{C_{i j}(t) \geq \hat{C}_{i j}(t) \mid \hat{h}_{i j}(t)\right\}\right)^{-1},
$$

then broadcasts a signal (of negligible duration). After the broadcast of the first signal, the contention procedure (of channel $j$ ) terminates and the corresponding user reports its CSI.

Once this procedure finishes, the contention of another channel gets started; clearly, two channels cannot have their contentions over the same period of time.

3) Transmission at time-slot t:

After getting the CSI (of time-slot $t-d$ ) of each channel, the BS uses these CSIs for transmission at time-slot $t$. It is obvious that the user that is selected to report its feedback (at time-slot $t-d$ ) of channel $j$ will also be the user transmitting over this channel (at time-slot $t$ ).

The main idea behind algorithm FSA is to approach the scheduling in the ideal system, while noting that the feedback decision is done by the users; the motivation behind letting the users be implicated in the decision was provided earlier. For each channel, this algorithm lets the users contend among each other in order to determine the user that will report its state, and this user will also be scheduled for transmission on the corresponding channel. As can be deduced from equation (13), for channel $j$ the user that is selected for feedback (and consequently for transmission on channel $j$ ), which results from the contention procedure, is the one that maximizes the following expression

$$
\hat{\tilde{q}}_{i}(t) \hat{C}_{i j}(t) \mathbb{P}\left\{C_{i j}(t) \geq \hat{C}_{i j}(t) \mid \hat{h}_{i j}(t)\right\},
$$

since, during the contention period, this user waits the smallest amount of time. The choice to maximize the above quantity can be explained as follows. First, recall that under the ideal system on channel $j$ the user that is scheduled for transmission is the one that maximizes $q_{i}(t) C_{i j}(t)$ (using the Max-Weight concept). Under system 'mdl', and accounting for delay $d$, the feedback decisions corresponding to the transmission of timeslot $t$ are made at time-slot $t-d$. The available information at user $i$ at time-slot $t-d$ includes all the queue lengths and in particular $\hat{\tilde{q}}_{i}(t)$, where we recall that $\hat{\tilde{q}}_{i}(t)=\tilde{q}(t-d)$, and $\hat{C}_{i j}(t)$ (which results from $\left.\hat{h}_{i j}(t)\right), \forall j$, with $\hat{C}_{i j}(t)=$ $C_{i j}(t-d)$. Based on the above and taking into account the outage possibility, which occurs when $\hat{C}_{i j}(t)>C_{i j}(t)$, it can be noticed that the best way to approximate the ideal scheduling on channel $j$ is by selecting the user that maximizes the quantity given in (14). 
1) Stability Performance Analysis for FSA: As mentioned earlier, the $L$ link rates are reported with a delay $d$. As a result of algorithm FSA, the expected weighted throughput, termed as $g_{\mathrm{mdl}}$, at time-slot $t$ is defined as follows

$$
\begin{aligned}
& g_{\text {mdl }}(\hat{\tilde{\mathbf{q}}}(t))= \\
& \mathbb{E}\left\{\sum_{j=1}^{L} \sum_{i=1}^{N} \hat{\tilde{q}}_{i}(t) \hat{C}_{i j}(t) \hat{Y}_{i j}(t) S_{i j}(t) \mathbb{1}_{\left(C_{i j}(t) \geq \hat{C}_{i j}(t)\right)} \mid \hat{\tilde{\mathbf{q}}}(t)\right\},
\end{aligned}
$$

in which $S_{i j}(t)$ represents the scheduling decision of link $(i j)$ at time-slot $t$. Thus, here $S_{i j}(t)=\hat{Y}_{i j}(t)$ and is equal to 1 if user $i$ is selected (at time $t-d$ ) by algorithm FSA to report its CSI (and consequently to transmit at time-slot $t$ ) and 0 otherwise. Recall that over each channel one and at most one channel is scheduled for transmission, i.e. $\sum_{i=1}^{N} S_{i j}(t) \leq 1$. We stress out that the indicator function $\mathbb{1}_{\left(C_{i j}(t) \geq \hat{C}_{i j}(t)\right)}$ is used because of a possible mismatch between the reported link state $\hat{C}_{i j}(t)$ and the actual link state $C_{i j}(t)$.

An alternative way to express $g_{\mathrm{mdl}}$ is by using the definition of $\mathcal{M}^{\mathrm{mdl}}(t)$ as

$$
\begin{aligned}
& g_{\text {mdl }}(\hat{\tilde{\mathbf{q}}}(t))= \\
& \mathbb{E}\left\{\sum_{(i j) \in \mathcal{M}^{\text {mdl }}(t)} \hat{\tilde{q}}_{i}(t) \hat{C}_{i j}(t) \mathbb{1}_{\left(C_{i j}(t) \geq \hat{C}_{i j}(t)\right)} \mid \hat{\tilde{\mathbf{q}}}(t)\right\} .
\end{aligned}
$$

We now investigate the stability performance of system 'mdl' by characterizing the minimum fraction that the stability region of algorithm FSA can achieve w.r.t. the stability region of the ideal system (i.e. 'pf'); clearly, this fraction is lower than 1 . To this end, we define $\eta$ as

$$
\eta=\max _{t,(i j)}\left\{\frac{C_{i j}(t)}{\hat{C}_{i j}(t)}\right\} .
$$

We here assume that all the possible values of $C_{i j}(t)$ (and thus of $\hat{C}_{i j}(t)$ ), given by $R_{1}, \ldots, R_{K}$, are different than zero. In practice, it is very rare not to be able to transmit at a non-zero rate. Moreover, we define $p_{\mathrm{c}}^{\min }$ as

$$
p_{\mathrm{c}}^{\min }=\min _{(i j)} p_{\mathrm{cin}}^{\min },
$$

with $p_{\mathrm{c} i j}^{\min }$ given by

$$
p_{\mathrm{c} i j}^{\min }=\min _{t, \hat{h}_{i j}(t)}\left\{\mathbb{P}\left\{C_{i j}(t) \geq \hat{C}_{i j}(t) \mid \hat{h}_{i j}(t)\right\}\right\},
$$

where obviously the value of $\hat{C}_{i j}(t)$ depends on $\hat{h}_{i j}(t)$. It is worth recalling that under system 'mdl' the scheduling decision at time-slot $t$ is based on the feedback decision made at time $t-d$. Also, recall that the BS broadcasts the queue lengths to the users each $T_{\mathrm{b}}$ slots. In addition, denote $\Lambda_{\mathrm{pf}}$ and $\Lambda_{\mathrm{mdl}}$ as the stability regions of systems 'pf' and 'mdl', respectively.

Based on the above, we now provide the stability region that system 'mdl' can achieve compared with the stability region of the ideal system.

Theorem 1. Algorithm FSA can achieve at least a fraction $\beta$ of the stability region achieved by the ideal system (with perfect and full feedback), i.e. the region $\Lambda_{\mathrm{mdl}}$ can be bounded as

$$
\beta \Lambda_{\mathrm{pf}} \subseteq \Lambda_{\mathrm{mdl}} \subseteq \Lambda_{\mathrm{pf}},
$$

where $\beta=\left(1-\frac{1}{T_{\mathrm{b}}}\right) \frac{p_{\mathrm{c}}^{\min }}{\eta}$.

Proof. We first provide an important result that will be useful for the rest of the proof. For this purpose, we define ' $\mathrm{pf}_{1}$ ' to be the system where the BS has perfect and full knowledge of the link states and then applies the Max-Weight rule for scheduling at time-slot $t$ using $\hat{\tilde{\mathbf{q}}}(t)$. Recall that $\hat{\tilde{q}}_{i}(t)=\tilde{q}_{i}(t-d)$, where $\tilde{q}_{i}(t)=q_{i}\left(n T_{\mathrm{b}}\right)$ for $t \in\left[n T_{\mathrm{b}},(n+1) T_{\mathrm{b}}[\right.$. We stress out that the only difference between ' $\mathrm{pf}_{1}$ ' and 'pf' (i.e. ideal system) is the fact that at time-slot $t$ the scheduling process (at the $\mathrm{BS}$ ) under ' $\mathrm{pf}_{1}$ ' will be done based on the queue lengths information $\hat{\tilde{\mathbf{q}}}(t)$, whereas under 'pf' the scheduling is based on $\mathbf{q}(t)$. Let $\Lambda_{\mathrm{pf}}$ and $\Lambda_{\mathrm{pf}_{1}}$ be the stability regions achieved under systems 'pf' and ' $\mathrm{pf}_{1}$, , respectively.

Based on the above, the following result can be stated.

Lemma 1. As long as $T_{\mathrm{b}}$ is finite, system ' $p f_{1}$ ' is strongly stable if and only if system 'pf' is strongly stable. That is to say, the following stability region result holds

$$
\Lambda_{\mathrm{pf}_{1}}=\Lambda_{\mathrm{pf}}
$$

Proof. The proof is similar to the proof in [24] and is thus omitted for sake of brevity.

From the above statement, it can be deduced that even if the scheduling is done based on an infrequent (i.e. outdated) queue lengths information, the stability of the system can still be achieved as long as the difference between the estimate (i.e. outdated value) and the exact value is bounded by a constant. It can be seen that this latter condition is satisfied for system ' $\mathrm{pf}_{1}$ ' since $T_{\mathrm{b}}$ is finite and the maximum per-time-slot length change in any queue is bounded (because the maximum arrival and service rates are bounded).

The rest of the proof consists of two main steps. We first show that $g_{\mathrm{mdl}}(\hat{\tilde{\mathbf{q}}}(t)) \geq \frac{p_{\mathrm{c}}^{\text {min }}}{\eta} g_{\mathrm{pf}_{1}}(\hat{\tilde{\mathbf{q}}}(t))$, where $g_{\mathrm{pf}_{1}}$ represents the expected weighted throughput under system ' $\mathrm{pf}_{1}$ ' and its expression can be found in Appendix A. We then demonstrate that the stability region achieved under 'mdl' reaches at least a fraction $\beta$ of the stability region achieved under ' pf $_{1}$ '. Based on these two steps and using the result in Lemma 1, we deduce the statement given in the theorem.

Please refer to Appendix A for the complete proof of the theorem.

It is important to highlight the fact that fraction $\beta$ just represents a lower bound on the performance guarantee of FSA, meaning that this algorithm may deliver better stability performance than that guaranteed by the lower bound. Based on the above observation and depending on the setting under consideration, we can state that FSA generally provides good stability performance. 


\section{B. Algorithm FMA and its Stability Performance}

In this section, we describe algorithm FMA and its implementation. Let 'dl' represent the system with delayed and limited feedback, and where the feedback and scheduling decisions are determined by algorithm FMA. For this system, we assume that the contention is done in discrete time. Under FMA, the feedback allocation is done at the users' side, and an amount of $F$ feedback resources is available. Specifically, under this algorithm, at time-slot $t-d$ the users decide to report at most $F$ link states; typically, $F$ should be greater than $L$. We assume that $\bar{F}$ link rates can be reported per time-slot. By neglecting the processing time at the user and the BS (in order to simplify the presentation), one can see that the time needed to report $F$ link rates is $\left\lceil F \times \bar{F}^{-1}\right\rceil$. We consider this time as the delay $d$ throughout this section; here $d$ is different from the delay in the previous section (in which $d$ is the fixed delay to report $L$ link states). In fact, one can see that the reported link states do not arrive at the BS at the same time. In order to simplify the presentation and the analysis/implementation, we consider in the feedback decision that the maximum value $d$ is the delay to report the link states to the BS. In addition, we consider that the feedback decision is done once each $d$ slots (the feedback decision is done per frame of $d$ slots) since the selected $F$ link states will take $d$ slots to be all reported. The scheduling will then use these reported $F$ link states during the next $d$ slots ( $d$ could be 1 according to the value of $F$ ). One can see clearly that by reporting more link states, the scheduling (in FMA) will have more states to be used in the Max-Weight scheduling but these reported states will be more outdated. There is hence a trade-off between reporting more link states and increasing the delay and reporting less link states that are less outdated. This trade-off will be explored numerically in Section IV. Recall that here we are considering memoryless scheduling and feedback decision in which the channel feedback and allocation do not consider the previous feedback decisions.

Let us denote the set of reported links by $\hat{\mathcal{F}}(t)$, where the cardinality $|\hat{\mathcal{F}}(t)| \leq F$. Then, due to the delay of $d$ slots, the BS receives the totality of this feedback at time-slot $t$. Let us define $F_{i}(t-d)$ to be the number of link states user $i$ decides at time $t-d$ to report under FMA, so we have $\sum_{i=1}^{N} F_{i}(t-d) \leq$ $F$. Also, we define $\alpha(t)$ to be some threshold that the system updates with the time; the update process will be provided later. To simplify the notation, we let $\hat{F}_{i}(t)$ and $\hat{\alpha}(t)$ represent $F_{i}(t-d)$ and $\alpha(t-d)$, respectively. Algorithm FMA is based on the idea of combining the Max-Weight and threshold-based concepts [20], [25]. It is worth mentioning that the steps in FMA are different from what is proposed in these latter works.

Remark 1. We want to draw attention to the fact that one can consider a model different from the one described here and therefore develop other feedback strategies. For instance, one can consider that the feedback is decided in each slot (and not each d slots as in FMA) and that the decision in each slot takes into account the reported links in the previous timeslots. This usually can be modeled as a Partially Observable Markov Decision Process (POMDP). However, the solutions of POMDP (or MDP) problems are known to be complex and may not be suitable for implementation in practice. This explains why we adopt in FMA a simple memoryless implementation in which the decision does not consider the past decisions but it takes into account the delay $d$.

Algorithm FMA: For system 'dl', the feedback and scheduling decisions are based on the following procedure.

1) Queue lengths broadcast every $T_{\mathrm{b}}$ slots:

Every $T_{\mathrm{b}}$ time-slots, that is to say, at time $0, T_{\mathrm{b}}, 2 T_{\mathrm{b}}, \ldots, n T_{\mathrm{b}}, \ldots$, the BS broadcasts the queue lengths of all users, where $T_{\mathrm{b}}$ is typically high. So each user has outdated knowledge of the state of its queue (and all other queues). Let $\tilde{q}_{i}(t)$ be the (outdated) queue length the users know at time $t$, i.e. $\tilde{q}_{i}(t)=q_{i}\left(n T_{\mathrm{b}}\right)$ for $t \in\left[n T_{\mathrm{b}},(n+1) T_{\mathrm{b}}[\right.$.

2) Queue lengths sorting at each user:

Each user knows then the queues of all other users and sorts all the queue lengths (including its queue) in a descending order. Let $i_{m}$ be the index of the user at the $m$ th position; e.g. $i_{1}$ is the index of the user with the largest queue length. A tie is broken by picking the user with the smallest index. We define $k\left(i_{m} j\right)$ as the state of link $\left(i_{m} j\right)$, so $\hat{C}_{i_{m} j}(t)=R_{k\left(i_{m} j\right)}$, where $k\left(i_{m} j\right) \in\{1, \ldots, K\}$.

3) Feedback decisions at time-slot $t-d$ :

To simplify the notation, we denote $\tilde{q}_{i}(t-d)$ as $\hat{\tilde{q}}_{i}(t)$. Set $\hat{F}_{i_{m}}(t)=0, \forall i_{m} \in\{1, \ldots, N\}$.

For $m=1$, which yields index $i_{1}$ and thus corresponds to the user with the largest queue length, the allocation process starts as the following:

a) For all $j \in\{1, \ldots, L\}$ :

- If there are enough feedback resources, i.e. if $\sum_{i_{m}=1}^{N} \hat{F}_{i_{m}}(t)<F$ :

* If channel $j$ satisfies the following inequality

$$
\begin{aligned}
& \hat{\tilde{q}}_{i_{m}}(t) \hat{C}_{i_{m} j}(t) \mathbb{P}\left\{C_{i_{m} j}(t) \geq \hat{C}_{i_{m} j}(t) \mid \hat{h}_{i_{m} j}(t)\right\} \\
& \geq \hat{\alpha}(t),
\end{aligned}
$$

then $\hat{C}_{i_{m} j}(t)$ will be reported to the BS and $\hat{F}_{i_{m}}(t)=\hat{F}_{i_{m}}(t)+1$.

* Else, $\hat{C}_{i_{m} j}(t)$ will not be reported.

- Otherwise, the allocation process stops since no feedback resources are available.

b) At this step user $i_{m}$ has just finished its part of the algorithm, so he broadcasts a special symbol to inform the BS that he finished the feedback allocation. Now, if the condition $\sum_{i_{m}=1}^{N} \hat{F}_{i_{m}}(t)<F$ is still satisfied and $m<N$, increment $m$ by 1 , and go to Step (a). Otherwise, the allocation process stops.

4) Scheduling decisions and transmission at time-slot $t$ : At time-slot $t$, the BS receives the link states that were selected at time-slot $t-d$. Then, for each channel, the BS applies the Max-Weight rule for scheduling. Specifically, over channel $j$ the BS schedules the user that results from 
the following

$$
\arg \max _{i:(i j) \in \hat{\mathcal{F}}(t)}\left\{q_{i}(t) \hat{C}_{i j}(t)\right\}
$$

where we recall that $\hat{\mathcal{F}}(t)$ is the set of reported link states and that if for some channel no feedback is reported, then no transmission can take place over this channel.

Roughly speaking, the idea behind the feedback decision approach in the proposed algorithm here is to allocate the feedback resources to the links which are more likely to be scheduled under the Max-Weight policy if all the link states are available at the BS. This is accomplished since the algorithm first allocates feedback resources to the user with the highest queue length if its Max-Weight product (see (22)) is high enough (i.e. above a threshold), then it moves to the next (in terms of largest queue length) user and so on. Hence, when the BS applies the Max-Weight rule for a given channel based on the subset of reported links states (resulting from the feedback decision under FMA), it will be very unlikely not to schedule the right user (which is the result of scheduling under a full CSI knowledge scenario).

It should be noted that even though we work under the assumption that the users estimate perfectly their fading coefficients, algorithms FSA and FMA can be adapted to the case where this estimation is not perfect. In this case, for both algorithms, $\hat{h}_{i j}(t)$ and $\hat{C}_{i j}(t)$ should be replaced by $\hat{\tilde{h}}_{i j}(t)$ and $\hat{\tilde{C}}_{i j}(t)$, respectively, where $\tilde{\tilde{h}}_{i j}(t)=\tilde{h}_{i j}(t-d)$ represents the (imperfectly) estimated fading coefficient at time-slot $t-d$ and $\hat{\tilde{C}}_{i j}(t)$ is the link rate calculated based on $\hat{\tilde{h}}_{i j}(t)$. Analyzing this case is interesting but is out of the scope of this work.

1) Stability Performance Analysis for FMA: We now investigate the stability performance of algorithm FMA. As mentioned earlier, this algorithm is proposed to imitate FSA which in its turn approaches the decisions of the ideal system. To analytically illustrate the good stability performance that FMA yields, we show that this algorithm imitates FSA to a great extent. For this purpose, we first discuss the conditions that an algorithm should guarantee in order to provide a good imitation of FSA.

In order to design an algorithm that greatly imitates FSA, two essential points should be accounted for. The first point is to ensure that over channel $j$ the user that will be scheduled is the result of

$$
\arg \max _{i}\left\{\hat{\tilde{q}}_{i}(t) \hat{C}_{i j}(t) \mathbb{P}\left\{C_{i j}(t) \geq \hat{C}_{i j}(t) \mid \hat{h}_{i j}(t)\right\}\right\} .
$$

The second point is to make sure that a transmission occurs over each channel, which can be ensured by having at least one reported link state for each channel. This is necessary since FSA guarantees a reported link state for each channel, leading to a transmission over all the channels. Based on these two conditions, the following remark can be made.

Remark 2. Algorithm FMA is designed in such a way as to imitate/approximate algorithm FSA as much as possible. Actually, how good this imitation/approximation is depends on several factors such as the amount of feedback resources
$F$ used by FMA, and the feedback limitation and imperfection, which is related to $F$ and to the velocity of each user. Expressed differently, FMA and FSA are not equivalent in terms of theoretical results, and FMA can be seen more as a heuristic algorithm without exact proofs of its capacity of approximating FSA.

The motivation behind the statement in Remark 2 is provided as follows.

By allocating a sufficient amount of feedback resources, $F$, with $F$ sufficiently large, and using condition (22) which ensures the selection (for the feedback) of links with large value of

$$
\hat{\tilde{q}}_{i}(t) \hat{C}_{i j}(t) \mathbb{P}\left\{C_{i j}(t) \geq \hat{C}_{i j}(t) \mid \hat{h}_{i j}(t)\right\},
$$

we can claim that algorithm FMA guarantees the first point (to ensure a good imitation of FSA) with high probability. On the other side, by a judicious choice of threshold $\hat{\alpha}(t)$ (or $\alpha(t)$ ), the second point (for a good imitation of FSA) can be guaranteed, that is to say, this threshold can be updated (with time) in such a way as to ensure that the probability of any one of the channels to have zero feedback is very low. We next explain how the threshold should be updated in order to guarantee the second point.

2) Threshold Update: We consider an approach under which the BS updates the threshold each $T_{\mathrm{u}}$ slots (e.g. at slots $\left.T_{\mathrm{u}}, 2 T_{\mathrm{u}}, 3 T_{\mathrm{u}}, \ldots\right)$ and then broadcasts it to the users, where the choice of $T_{\mathrm{u}}$ depends on the system under consideration. For notational convenience, suppose that $t_{\mathrm{u}}-d$ is the timeslot at which the BS has to calculate the threshold, i.e. $t_{\mathrm{u}}-d=n T_{\mathrm{u}}$ for some non-negative integer $n$. Hence, at time-slot $t_{\mathrm{u}}-d$ the BS calculates threshold $\hat{\alpha}\left(t_{\mathrm{u}}\right)$, where we recall that $\hat{\alpha}\left(t_{\mathrm{u}}\right)=\alpha\left(t_{\mathrm{u}}-d\right)$. Note that at this slot the BS has complete knowledge of the queue lengths of this slot but knows nothing about the link states of this slot; recall that these lengths and states are denoted by $\hat{q}_{i j}\left(t_{\mathrm{u}}\right)$ and $\hat{C}_{i j}\left(t_{\mathrm{u}}\right)$, respectively. Further, a reasonable assumption that can be made here is that the BS knows the probabilities $\mathbb{P}\left\{C_{i j}\left(t_{\mathrm{u}}\right) \geq \hat{C}_{i j}\left(t_{\mathrm{u}}\right) \mid \hat{C}_{i j}\left(t_{\mathrm{u}}\right)=R_{k}\right\}$. As indicated earlier, the threshold should ensure that it is very likely that every channel gets at least one feedback, or equivalently, that it is very unlikely that a channel gets zero feedback.

Under algorithm FMA, a necessary condition for link $(i j)$ to get selected for the feedback at time-slot $t_{\mathrm{u}}-d$ is to have (see equation (22))

$$
\hat{\tilde{q}}_{i}\left(t_{\mathrm{u}}\right) \hat{C}_{i j}\left(t_{\mathrm{u}}\right) \mathbb{P}\left\{C_{i j}\left(t_{\mathrm{u}}\right) \geq \hat{C}_{i j}\left(t_{\mathrm{u}}\right) \mid \hat{h}_{i j}\left(t_{\mathrm{u}}\right)\right\} \geq \hat{\alpha}\left(t_{\mathrm{u}}\right) .
$$

Based on this observation and the available information at the $\mathrm{BS}$, the necessary condition for channel $j$ to get at least one feedback can be approximated by the BS as

$$
\sum_{i} \sum_{k: \hat{q}_{i}\left(t_{\mathrm{u}}\right) R_{k} \mathbb{P}\left\{C_{i j}\left(t_{\mathrm{u}}\right) \geq \hat{C}_{i j}\left(t_{\mathrm{u}}\right) \mid \hat{C}_{i j}\left(t_{\mathrm{u}}\right)=R_{k}\right\} \geq \hat{\alpha}\left(t_{\mathrm{u}}\right)} p_{i j k} \geq 1,
$$

where $p_{i j k}$ is the probability that link $(i j)$ is at state $k$, which is supposed to be known by the BS. Define $\Omega_{i j k}$ and $\omega_{j}$ as

$$
\Omega_{i j k}=\hat{q}_{i}\left(t_{\mathrm{u}}\right) R_{k} \mathbb{P}\left\{C_{i j}\left(t_{\mathrm{u}}\right) \geq \hat{C}_{i j}\left(t_{\mathrm{u}}\right) \mid \hat{C}_{i j}\left(t_{\mathrm{u}}\right)=R_{k}\right\},
$$




$$
\omega_{j}=\sum_{i} \sum_{k: \Omega_{i j k} \geq \hat{\alpha}\left(t_{\mathrm{u}}\right)} p_{i j k} .
$$

Hence, a necessary condition for every channel to get at least one feedback can be stated as

$$
\omega_{j} \geq 1, \forall j .
$$

The following constraint should be added when calculating $\hat{\alpha}\left(t_{\mathrm{u}}\right)$

$$
\sum_{j} \omega_{j} \leq F \text { and as close as possible to } F .
$$

The conditions in (31) ensure that the average number of links that are eligible to report their states (see (26)), which is given by $\sum_{j} \omega_{j}$, is pushed as close as possible to $F$. This is important due to the following reasons: (a) at most $F$ (with $F>L$ ) link states can be reported, due to an amount of feedback resources equal to $F$, (b) the more the number of links that are eligible for the feedback is greater than $F$, the more likely it is that algorithm FMA does not report the best links in terms of maximizing the product $\hat{\tilde{q}}_{i}\left(t_{\mathrm{u}}\right) \hat{C}_{i j}\left(t_{\mathrm{u}}\right) \mathbb{P}\left\{C_{i j}\left(t_{\mathrm{u}}\right) \geq \hat{C}_{i j}\left(t_{\mathrm{u}}\right) \mid \hat{h}_{i j}\left(t_{\mathrm{u}}\right)\right\}$, and (c) the more the number of links that are eligible for the feedback is lower than $F$, the less the feedback resources are exploited efficiently, which may lead to one or multiple channels with zero feedback. Note that the lower the threshold is, the greater $w_{j}$ and $\sum_{j} w_{j}$ will be.

Based on all the above, the following procedure can be adopted to compute $\hat{\alpha}\left(t_{\mathrm{u}}\right)$ :

1. The BS chooses any threshold value such that, using this threshold, the condition $\omega_{j} \geq 1$ is not satisfied for all $j$. Denote this value by $\hat{\alpha}_{0}\left(t_{\mathrm{u}}\right)$.

2. Then, starting from $\hat{\alpha}_{0}\left(t_{\mathrm{u}}\right)$, it decreases the threshold value until the condition $\omega_{j} \geq 1$ is satisfied for all $j$. Denote the threshold at this stage by $\hat{\alpha}_{1}\left(t_{\mathrm{u}}\right)$.

3. If using $\hat{\alpha}_{1}\left(t_{\mathrm{u}}\right)$ we have $\sum_{j} \omega_{j}<F$, then the BS decreases the threshold value (starting from $\hat{\alpha}_{1}\left(t_{\mathrm{u}}\right)$ ) until having $\sum_{j} \omega_{j}$ as close as possible to $F$ and such that $\sum_{j} \omega_{j} \leq F$.

Finally, we point out that if the BS knows

$$
\mathbb{E}_{\hat{h}_{i j}\left(t_{\mathrm{u}}\right)}\left\{\mathbb{P}\left\{C_{i j}\left(t_{\mathrm{u}}\right) \geq \hat{C}_{i j}\left(t_{\mathrm{u}}\right) \mid \hat{C}_{i j}\left(t_{\mathrm{u}}\right)=R_{k}, \hat{h}_{i j}\left(t_{\mathrm{u}}\right)\right\}\right\},
$$

where we recall that $\hat{h}_{i j}\left(t_{\mathrm{u}}\right)=h_{i j}\left(t_{\mathrm{u}}-d\right)$, then, clearly, it is better to define $\Omega_{i j k}$ as

$$
\begin{aligned}
& \Omega_{i j k}=\hat{q}_{i}\left(t_{\mathrm{u}}\right) R_{k} \times \\
& \mathbb{E}_{\hat{h}_{i j}\left(t_{\mathrm{u}}\right)}\left\{\mathbb{P}\left\{C_{i j}\left(t_{\mathrm{u}}\right) \geq \hat{C}_{i j}\left(t_{\mathrm{u}}\right) \mid \hat{C}_{i j}\left(t_{\mathrm{u}}\right)=R_{k}, \hat{h}_{i j}\left(t_{\mathrm{u}}\right)\right\}\right\} .
\end{aligned}
$$

Taking this new definition into account, the same procedure as before can be used to find $\hat{\alpha}\left(t_{\mathrm{u}}\right)$.

\section{Numerical Results}

\begin{tabular}{|c|c|c|}
\hline & Parameter & Description \\
\hline & $N$ & Number of users \\
\hline & $L$ & Number of channels \\
\hline & $\gamma_{i j}(t)$ & SNR of link $(i j)$ \\
\hline & $C_{i j}(t)$ & State of link $(i j)$ \\
\hline & $A_{i}(t)$ & Arrival rate of user $i$ \\
\hline & $a_{i}$ & Mean arrival rate of user $i$ \\
\hline Parameter & \multicolumn{2}{|r|}{ Description } \\
\hline$q_{i}(t)$ & \multicolumn{2}{|r|}{ Queue length of user $i$} \\
\hline$d$ & \multicolumn{2}{|r|}{ Delay in the feedback process } \\
\hline$F$ & \multicolumn{2}{|c|}{ Amount of feedback that can be reported per slot } \\
\hline$F$ & \multicolumn{2}{|c|}{ Maximum amount of feedback under FMA } \\
\hline$\hat{Y}_{i j}(t)$ & \multicolumn{2}{|c|}{ Feedback decision for link $(i j)$ at time-slot $t-d$} \\
\hline$S_{i j}(t)$ & \multicolumn{2}{|c|}{ Scheduling decision for link $(i j)$ at time-slot $t$} \\
\hline
\end{tabular}

We set $N=L=30$ (unless stated otherwise), $P=$ $10 \log _{10}(10)=10 \mathrm{~dB}$. We consider an LTE-like system with a bandwidth of $180 \mathrm{KHz}$ per channel and a carrier frequency $f_{\mathrm{c}}=2.1 \mathrm{GHz}$. Let $T_{\mathrm{s}}$ represent the slot period, which is set to
Table I: List of the main parameters and their descriptions

Table II: Possible rates used for the simulations

\begin{tabular}{|l||l|l|l|l|l|} 
Rate (bits/slot) & 505.32 & 570.58 & 622.69 & 666.08 & 703.24 \\
\hline Rate (bits/slot) & 735.74 & 764.62 & 790.61 & 891.75 & 964.36
\end{tabular}

\begin{tabular}{|l||c|c|c|c|c|}
\hline Rate (bits/slot) & 735.74 & 764.62 & 790.61 & 891.75 & 964.36
\end{tabular}

1 msec. Assume that the $\sigma_{\mathrm{z}, i j}$ are all equal to 1 , i.e. $\sigma_{\mathrm{z}, i j}=1$, $\forall i, j$. To model the impact of delay, we consider the GaussMarkov block fading process [26]. Based on this model, we can write

$$
h_{i j}(t)=\sigma \hat{h}_{i j}(t)+e_{i j}(t)
$$

where $h_{i j}(t)$ is a complex normal random variable with zero mean and unit variance, i.e. $h_{i j}(t) \sim \mathcal{C N}(0,1)$, and where $e_{i j}(t) \sim \mathcal{C N}\left(0, \sigma_{\mathrm{e}}^{2}\right)$ is the error due to delay. Notice that $\hat{h}_{i j}(t) \sim \mathcal{C N}(0,1)$ and $\sigma_{\mathrm{e}}^{2}=1-\sigma^{2}$. The correlation coefficient is given by $\sigma=J_{0}\left(2 \pi f_{\mathrm{ds}} T_{\mathrm{s}} d\right)$, with Doppler spread $f_{\mathrm{ds}}$, where $T_{\mathrm{S}}$ is the slot duration, $d$ is the delay in number of time-slots, and $J_{0}(\cdot)$ is the zero-th order Bessel function of the first kind. The Doppler spread can be given by $f_{\mathrm{ds}}=\frac{f_{\mathrm{c}} v}{c}$, where $v$ is the user velocity and $c=3 \times 10^{8} \mathrm{~m} / \mathrm{sec}$ is the speed of light; we assume that all the users have the same velocity $v$. Hence, the correlation coefficient $\sigma=J_{0}\left(2 \pi f_{\mathrm{ds}} T_{\mathrm{s}} d\right)=J_{0}\left(2 \pi \frac{f_{\mathrm{c} v} v}{c} T_{\mathrm{s}} d\right)$. The fading coefficients are assumed to be i.i.d. across users and frequencies. The set of possible rates $\left\{R_{1}, \ldots, R_{K}\right\}$ is given in Table II. We suppose that all the users have Poisson incoming traffic with the same mean arrival rate $a$, i.e. $a_{i}=a$, $\forall i \in\{1, \ldots, N\}$.

To show the stability performance of the system, we plot the total average queue length, defined as $\frac{1}{M_{\mathrm{s}}} \sum_{t=0}^{M_{\mathrm{s}}-1} \sum_{k=1}^{N} q_{k}(t)$, for different values of mean arrival rate $a$, where $M_{\mathrm{s}}$ represents the number of time-slots each simulation lasts (per mean arrival rate). We set $M_{\mathrm{s}}=10^{4}$. Note that the point where the total average queue length begins to increase very steeply is the point where the system becomes unstable. It is worth mentioning that the best representation of the stability performance of an algorithm is the stability region this algorithm can achieve. However, in our case, this region is an $N$-dimensional polytope, and since we are interested in a system where there is a large number of users, characterizing and plotting this region is not possible. This explains our choice of the total average queue length as an alternative performance metric. As alluded earlier, for a fixed mean arrival rate vector, which represents a direction from the origin, this metric allows us to detect the boundary of the stability region in this direction. 


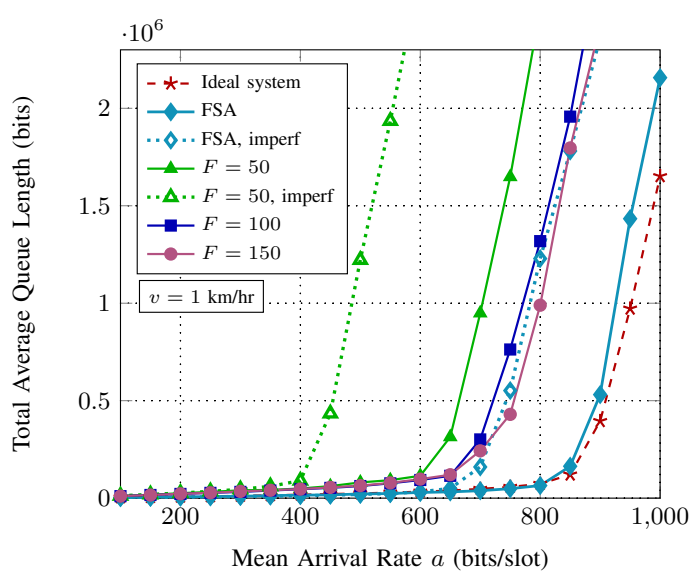

Figure 2: Total average queue length vs. mean arrival rate $a$. Here, the velocity $v=1 \mathrm{~km} / \mathrm{hr}$

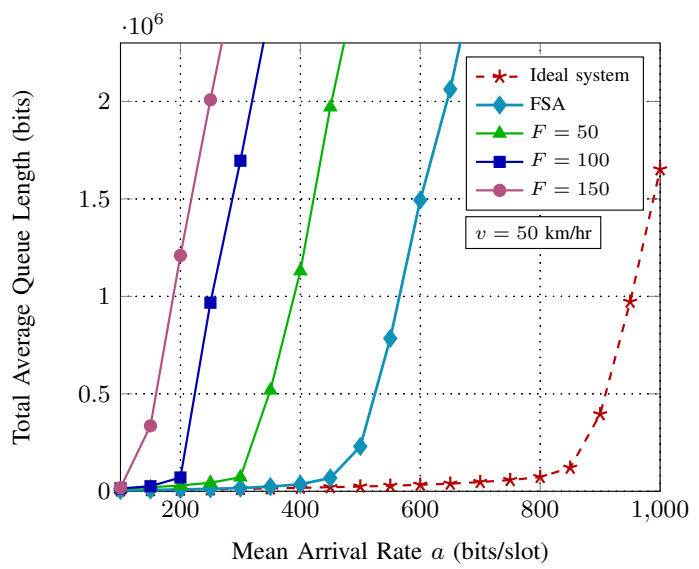

Figure 3: Total average queue length vs. mean arrival rate $a$. Here, the velocity $v=50 \mathrm{~km} / \mathrm{hr}$.

We first provide two figures where in each one we consider a different value of the velocity, $v$. Specifically, in Figures 2 and 3 we set $v=1$ and $50 \mathrm{~km} / \mathrm{hr}$, respectively. Each figure depicts the variation of the total average queue length for different values of the mean arrival rate under various system settings. The first setting is ideal system 'pf', i.e. system with perfect and full feedback. The second setting is system 'mdl', i.e. system with delayed and limited feedback where algorithm FSA is used for feedback and scheduling decisions. The three remaining settings consider system 'dl', i.e. system with delayed and limited feedback where algorithm FMA is used for feedback and scheduling decisions, for three different amounts of feedback resources $F=50,100$, and 150. Recall that delay $d$ depends on the value of $F$. Here, we assume that $F=50,100$, and 150 result in $d=1,2$, and 3 slots, respectively.

Starting by the performance of FMA, the simulations show that for relatively small values of the velocity (see Figure 2 ), increasing the feedback resources $F$ to some limit can provide better stability performance. This is due to the fact that for small values of $v$, the variance of the error given by $\sigma_{\mathrm{e}}^{2}=1-\sigma^{2}$ is sufficiently small, so the gain coming from having more feedback information overcomes the loss due to delay. Evidently, the limit until which if we increase $F$ we get better performance depends on $\sigma_{\mathrm{e}}$ and consequently on $v$. Recall that FMA and the scheduling are memoryless and do not consider the previous feedback decisions, which explains in part this behavior. For instance, for $v=1 \mathrm{~km} / \mathrm{hr}$ the best $F$ we can choose is 150 . If we keep increasing $v$, there will be no gain from taking $F>50$. This can be clearly seen in Figure 3. Specifically, for $v=50 \mathrm{~km} / \mathrm{hr}, F=50$ yields better performance than both of $F=100$ and $F=150$ cases. This is due to the fact that for relatively high velocities the variance of the delay error, i.e. $\sigma_{\mathrm{e}}^{2}$, is sufficiently high, so the loss due to delay is bigger than the gain coming from having more feedback information.

As for the performance of FSA, it can be easily noticed that the lesser the delay error is (i.e. lower $v$ ), the closer the performance of algorithm FSA is w.r.t. the ideal system.

In Figure 2, for each of FSA and (FMA) $F=50$, in addition to the delayed- and limited-feedback case, we also simulate the case where there is an imperfection in the channel estimation. As explained in Section III, FSA and FMA can be easily adapted to this case. We adopt a classical imperfection model under which the relation between the effective fading coefficient, $h_{i j}$, and the estimated one, $\tilde{h}_{i j}$, is as follows: $h_{i j}=\tilde{h}_{i j}+e_{1, i j}$, where $e_{1, i j}$ represents the estimation error. For the simulations, the standard deviation of this error is set to 0.2 . Note that the delay model is still given by (34) but the channel at time $t-d$ will be replaced by the estimated channel (instead of the effective one).

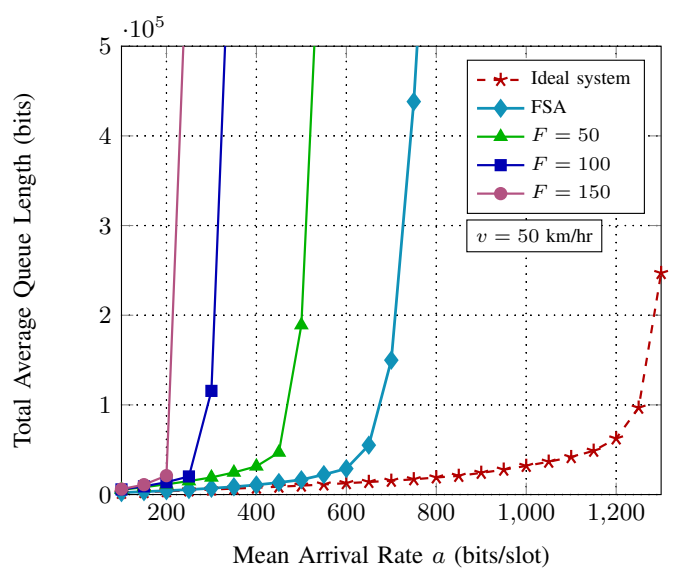

Figure 4: Total average queue length vs. mean arrival rate $a$. Here, $N=20$ users and $v=50 \mathrm{~km} / \mathrm{hr}$.

Figure 4 considers settings similar to those used in Figure 3 except that the number of users, $N$ is reduced (from 30 ) to 20 . It can be seen that, in this case, the stability performance improves for each of systems 'dl', 'mdl' and 'pf' compared with the case where $N=30$. This is expected since, for a fixed number of channels, a smaller number of users in the system leads to more scheduling/serving opportunities for these users.

In Figure 5, we compare our proposed schemes with a baseline scheme, termed BLA, for the case where $v=5 \mathrm{~km} / \mathrm{hr}$ and $N=30$. For the scheduling mechanism under BLA, we adopt the same approach as under FMA (and FSA), i.e. the Max-Weight rule. By considering this rule, we are sure that the baseline scheme has the best possible scheduling approach [9]. 


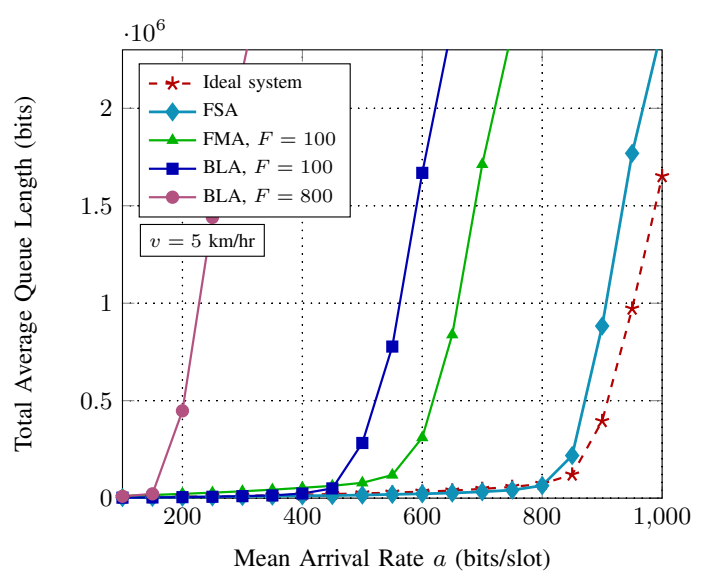

Figure 5: Total average queue length vs. mean arrival rate $a$. Here, $v=5$ $\mathrm{km} / \mathrm{hr}$.

For this scheme, we consider periodic CSI feedback, which is the concept used in LTE [2]. The periodicity here is translated by adopting a round-robin approach over all the users and channels, where the amount of feedback resources per period is denoted $F$. From Figure 5, it is clear that our proposed approaches yield better stability performance than the baseline scheme.

\section{CONCLUSION}

In this paper, we addressed the problems of feedback allocation and scheduling for a multiuser multichannel downlink cellular network under limited and delayed feedback. We first proposed an efficient joint feedback allocation and scheduling algorithm, in which the decisions are made at the users' side. This algorithm is shown to achieve good stability performance w.r.t. the ideal system, however it is suitable for a continuoustime contention scheme. In case the contention is only possible in discrete time, we proposed a second algorithm, which uses a threshold-based concept, that imitates the first one as much as possible and thus guarantees good stability performance. For this algorithm, the feedback decision is also done at the users' side, and then the BS uses this feedback to perform users scheduling. Regarding the choice of the amount of feedback resources for this algorithm, we provided numerical results that find the best feedback period; these results are given for various system setups, i.e. different values of users velocity. Finally, using simulations, we validated that the proposed algorithms provide good stability performance.

\section{APPENDIX A \\ PROOF OF THEOREM 1}

The proof consists of two main steps. We first show that $g_{\text {mdl }}(\hat{\tilde{\mathbf{q}}}(t)) \geq \frac{p_{\mathrm{c}}^{\min }}{\eta} g_{\mathrm{pf}_{1}}(\hat{\tilde{\mathbf{q}}}(t))$, where $g_{\mathrm{pf}_{1}}$ is defined later in the proof. After that, using this inequality, we demonstrate that the stability region achieved under 'mdl' (using FSA) reaches at least a fraction $\beta$ of the stability region achieved under 'pf ${ }_{1}$ '. Finally, based on these two steps and using the result in Lemma 1, we deduce the statement given in the theorem.

- Step 1: Here, we prove that $g_{\text {mdl }}(\hat{\tilde{\mathbf{q}}}(t)) \geq \frac{p_{\mathrm{c}}^{\min }}{\eta} g_{\mathrm{pf}_{1}}(\hat{\tilde{\mathbf{q}}}(t))$. To this end, we define ' $m p f_{1}$ ' to be the system with full but delayed CSI (i.e. delay of $d$ slots) at the BS, and where at timeslot $t$ the Max-Weigh policy is used for scheduling based on $\hat{\tilde{\mathbf{q}}}(t)$. Under this system, the expected weighted throughput, termed $g_{\text {mpf }_{1}}$, can be expressed as

$$
\begin{aligned}
& g_{\text {mpf }_{1}}(\hat{\tilde{\mathbf{q}}}(t))= \\
& \mathbb{E}\left\{\sum_{j=1}^{L} \sum_{i=1}^{N} \hat{\tilde{q}}_{i}(t) \hat{C}_{i j}(t) S_{i j}(t) \mathbb{1}_{\left(C_{i j}(t) \geq \hat{C}_{i j}(t)\right)} \mid \hat{\tilde{\mathbf{q}}}(t)\right\} .
\end{aligned}
$$

We first show that $g_{\text {mpf }_{1}}(\hat{\tilde{\mathbf{q}}}(t)) \geq \frac{p_{\mathrm{c}}^{\min }}{\eta} g_{\mathrm{pf}_{1}}(\hat{\tilde{\mathbf{q}}}(t))$. It can be easily seen that under 'pf ${ }_{1}$ ' the expected weighted throughput, termed as $g_{\mathrm{pf}_{1}}$, can be given by

$$
g_{\mathrm{pf}_{1}}(\hat{\tilde{\mathbf{q}}}(t))=\mathbb{E}\left\{\sum_{j=1}^{L} \sum_{i=1}^{N} \hat{\tilde{q}}_{i}(t) C_{i j}(t) S_{i j}(t) \mid \hat{\tilde{\mathbf{q}}}(t)\right\} .
$$

Expected weighted throughputs $g_{\mathrm{pf}_{1}}(\hat{\tilde{\mathbf{q}}}(t))$ and $g_{\text {mpf }_{1}}(\hat{\tilde{\mathbf{q}}}(t))$ can be re-expressed as the following

$$
\begin{aligned}
& g_{\mathrm{pf}_{1}}(\hat{\tilde{\mathbf{q}}}(t))=\mathbb{E}\left\{\sum_{(i j) \in \mathcal{M}^{\mathrm{p}_{1}}(t)} \hat{\tilde{q}}_{i}(t) C_{i j}(t) \mid \hat{\tilde{\mathbf{q}}}(t)\right\}, \\
& g_{\text {mpf }_{1}}(\hat{\tilde{\mathbf{q}}}(t))= \\
& \mathbb{E}\left\{\sum_{(i j) \in \mathcal{M}^{\text {mpf }_{1}}(t)} \hat{\tilde{q}}_{i}(t) \hat{C}_{i j}(t) \mathbb{1}_{\left(C_{i j}(t) \geq \hat{C}_{i j}(t)\right)} \mid \hat{\tilde{\mathbf{q}}}(t)\right\},
\end{aligned}
$$

where $\mathcal{M}^{\mathrm{pf}_{1}}(t)$ and $\mathcal{M}^{\mathrm{mpf}_{1}}(t)$ stand for the subsets of scheduled users under ' $\mathrm{pf}_{1}$ ' and ' $\mathrm{mpf}_{1}$ ', respectively, at time-slot $t$. Let $\mathbf{h}$ be a vector that represents the fading of all the links at time-slot $t$. We also define $\hat{\mathbf{h}}$ to be the fading of these links at time-slot $t-d$. Then, we can write

$$
\begin{aligned}
& g_{\text {mpf }_{1}}(\hat{\tilde{\mathbf{q}}}(t)) \\
& =\mathbb{E}_{\hat{\mathbf{h}}}\left\{\mathbb{E}_{\mathbf{h} \mid \hat{\mathbf{h}}}\left\{\sum_{(i j) \in \mathcal{M}^{\text {mpf }_{1}(t)}} \hat{\tilde{q}}_{i}(t) \hat{C}_{i j}(t) \mathbb{1}_{\left(C_{i j}(t) \geq \hat{C}_{i j}(t)\right)} \mid \hat{\tilde{\mathbf{q}}}(t)\right\}\right\} \\
& =\mathbb{E}_{\hat{\mathbf{h}}}\left\{\sum_{(i j) \in \mathcal{M}^{\text {mp }_{1}}(t)} \hat{\tilde{\tilde{f}}}_{i}(t) \hat{C}_{i j}(t) \mathbb{P}\left\{C_{i j}(t) \geq \hat{C}_{i j}(t) \mid \hat{h}_{i j}(t)\right\} \mid \hat{\tilde{\mathbf{q}}}(t)\right\} .
\end{aligned}
$$

Let us define $p_{\mathrm{c}}^{\min }$ as $p_{\mathrm{c}}^{\min }=\min _{(i j)} p_{\mathrm{c} i j}^{\min }$, with $p_{\mathrm{c} i j}^{\min }=$ $\min _{t, \hat{h}_{i j}(t)}\left\{\mathbb{P}\left\{C_{i j}(t) \geq \hat{C}_{i j}(t) \mid \hat{h}_{i j}(t)\right\}\right\}$, where we recall that the value of $\hat{C}_{i j}(t)$ depends on $\hat{h}_{i j}(t)$. Based on the expression of $g_{\text {mpf }_{1}}(\hat{\tilde{\mathbf{q}}}(t))$ and the definition of $p_{\mathrm{c}}^{\min }$, we get

$$
\mathbb{E}\left\{\sum_{(i j) \in \mathcal{M}^{\text {mpf }_{1}}(t)} \hat{\tilde{q}}_{i}(t) \hat{C}_{i j}(t) \mid \hat{\tilde{\mathbf{q}}}(t)\right\} \leq \frac{g_{\mathrm{mpf}_{1}}(\hat{\tilde{\mathbf{q}}}(t))}{p_{\mathrm{c}}^{\min }} .
$$

By defining $\eta$ as $\eta=\max _{t,(i j)}\left\{\frac{C_{i j}(t)}{\hat{C}_{i j}(t)}\right\}$, we obtain the following inequality

$$
\begin{aligned}
& \mathbb{E}\left\{\sum_{(i j) \in \mathcal{M}^{\mathrm{pf}_{1}}(t)} \hat{\tilde{q}}_{i}(t) C_{i j}(t) \mid \hat{\tilde{\mathbf{q}}}(t)\right\} \leq \\
& \eta \mathbb{E}\left\{\sum_{(i j) \in \mathcal{M}^{\text {mf }_{1}}(t)} \hat{\tilde{q}}_{i}(t) \hat{C}_{i j}(t) \mid \hat{\tilde{\mathbf{q}}}(t)\right\} .
\end{aligned}
$$


This inequality can be proved as follows. Let $i_{1}$ and $i_{2}$ be the scheduled users over channel $j$ under systems ' $\mathrm{pf}_{1}$ ' and 'mpf ${ }_{1}$ ', respectively. These users are selected according to the following

$$
i_{1}=\arg \max _{i}\left\{\hat{\tilde{q}}_{i}(t) C_{i j}(t)\right\}, \quad i_{2}=\arg \max _{i}\left\{\hat{\tilde{q}}_{i}(t) \hat{C}_{i j}(t)\right\} .
$$

Based on the above and the definition of $\eta$, we get

$$
\hat{\tilde{q}}_{i_{1}}(t) C_{i_{1} j}(t) \leq \hat{\tilde{q}}_{i_{1}}(t) \hat{C}_{i_{1} j}(t) \eta \leq \hat{\tilde{q}}_{i_{2}}(t) \hat{C}_{i_{2} j}(t) \eta .
$$

Hence, the inequality in (41) follows by summing over all the channels $j \in\{1, \ldots, L\}$. Combining (40) and (41) yields $g_{\text {mpf }_{1}}(\hat{\tilde{\mathbf{q}}}(t)) \geq \frac{p_{\mathrm{c}}^{\min }}{\eta} g_{\mathrm{pf}_{1}}(\hat{\tilde{\mathbf{q}}}(t))$. Now, we show that $g_{\mathrm{mdl}}(\hat{\tilde{\mathbf{q}}}(t)) \geq$ $g_{\text {mpf }_{1}}(\hat{\tilde{\mathbf{q}}}(t))$. By denoting $\mathcal{M}^{\mathrm{mdl}}(t)$ as the subset of users scheduled for transmission under 'mdl' at time-slot $t$, we can rewrite $g_{\text {mdl }}(\hat{\tilde{\mathbf{q}}}(t))$ as

$$
\begin{aligned}
& g_{\mathrm{mdl}}(\hat{\tilde{\mathbf{q}}}(t))= \\
& \mathbb{E}\left\{\sum_{(i j) \in \mathcal{M}^{\mathrm{mdl}}(t)} \hat{\tilde{q}}_{i}(t) \hat{C}_{i j}(t) \mathbb{1}_{\left(C_{i j}(t) \geq \hat{C}_{i j}(t)\right)} \mid \hat{\tilde{\mathbf{q}}}(t)\right\} .
\end{aligned}
$$

We define $g_{\mathrm{mdl}, j}(\hat{\tilde{\mathbf{q}}}(t))$ and $g_{\mathrm{mpf}_{1}, j}(\hat{\tilde{\mathbf{q}}}(t))$ as the following

$$
\begin{aligned}
& g_{\mathrm{mdl}, j}(\mathbf{q}(t))=\mathbb{E}\left\{\hat{\tilde{q}}_{i_{1}}(t) \hat{C}_{i_{1} j}(t) \mathbb{1}_{\left(C_{i_{1} j}(t) \geq \hat{C}_{i_{1} j}(t)\right)} \mid \hat{\tilde{\mathbf{q}}}(t)\right\}, \\
& g_{\operatorname{mpf}_{1}, j}(\hat{\tilde{\mathbf{q}}}(t))=\mathbb{E}\left\{\hat{\tilde{q}}_{i_{\mathrm{f}}}(t) \hat{C}_{i_{\mathrm{f}} j}(t) \mathbb{1}_{\left(C_{i_{\mathrm{f}} j}(t) \geq \hat{C}_{i_{\mathrm{f}} j}(t)\right)} \mid \hat{\tilde{\mathbf{q}}}(t)\right\},
\end{aligned}
$$

where $i_{1}$ and $i_{\mathrm{f}}$ denote the scheduled users over channel $j$ under 'mdl' and ' $\mathrm{mpf}_{1}$ ', respectively. These users can be determined according to the following

$$
\begin{gathered}
i_{1}=\arg \max _{i}\left\{\hat{\tilde{q}}_{i}(t) \hat{C}_{i j}(t) \mathbb{P}\left\{C_{i j}(t) \geq \hat{C}_{i j}(t) \mid \hat{h}_{i j}(t)\right\}\right\}, \\
i_{\mathrm{f}}=\arg \max _{i}\left\{\hat{\tilde{q}}_{i}(t) \hat{C}_{i j}(t)\right\} .
\end{gathered}
$$

Based on the above, it can be seen that

$$
\begin{aligned}
& \hat{\tilde{q}}_{i_{1}}(t) \hat{C}_{i_{1} j}(t) \mathbb{P}\left\{C_{i_{1} j}(t) \geq \hat{C}_{i_{1} j}(t) \mid \hat{h}_{i_{1} j}(t)\right\} \geq \\
& \hat{\tilde{q}}_{i_{\mathrm{f}}}(t) \hat{C}_{i_{\mathrm{f}} j}(t) \mathbb{P}\left\{C_{i_{\mathrm{f}} j}(t) \geq \hat{C}_{i_{\mathrm{f}} j}(t) \mid \hat{h}_{i_{\mathrm{f}} j}(t)\right\} .
\end{aligned}
$$

Hence, we get

$$
\begin{aligned}
& g_{\mathrm{mpf}_{1}, j}(\hat{\tilde{\mathbf{q}}}(t)) \\
& =\mathbb{E}_{\hat{\mathbf{h}}}\left\{\mathbb{E}_{\mathbf{h} \mid \hat{\mathbf{h}}}\left\{\max _{i}\left\{\hat{\tilde{q}}_{i}(t) \hat{C}_{i j}(t)\right\} \mathbb{1}_{\left(C_{i j}(t) \geq \hat{C}_{i j}(t)\right)} \mid \hat{\tilde{\mathbf{q}}}(t)\right\}\right\} \\
& =\mathbb{E}_{\hat{\mathbf{h}}}\left\{\mathbb{E}_{\mathbf{h} \mid \hat{\mathbf{h}}}\left\{\hat{\tilde{q}}_{i_{\mathrm{f}}}(t) \hat{C}_{i_{\mathrm{f}} j}(t) \mathbb{1}_{\left(C_{i_{\mathrm{f}} j}(t) \geq \hat{C}_{i_{\mathrm{f}} j}(t)\right)} \mid \hat{\tilde{\mathbf{q}}}(t)\right\}\right\} \\
& =\mathbb{E}_{\hat{\mathbf{h}}}\left\{\hat{\tilde{q}}_{i_{\mathrm{f}}}(t) \hat{C}_{i_{\mathrm{f}} j}(t) \mathbb{P}\left\{C_{i_{\mathrm{f}} j}(t) \geq \hat{C}_{i_{\mathrm{f}} j}(t) \mid \hat{h}_{i_{\mathrm{f}} j}(t)\right\} \mid \hat{\tilde{\mathbf{q}}}(t)\right\} \\
& \stackrel{(a)}{\leq} \mathbb{E}_{\hat{\mathbf{h}}}\left\{\hat{\tilde{q}}_{i_{1}}(t) \hat{C}_{i_{1} j}(t) \mathbb{P}\left\{C_{i_{1} j}(t) \geq \hat{C}_{i_{1} j}(t) \mid \hat{h}_{i_{1} j}(t)\right\} \mid \hat{\tilde{\mathbf{q}}}(t)\right\} \\
& =\mathbb{E}_{\hat{\mathbf{h}}}\left\{\mathbb{E}_{\mathbf{h} \mid \hat{\mathbf{h}}}\left\{\hat{\tilde{q}}_{i_{1}}(t) \hat{C}_{i_{1} j}(t) \mathbb{1}_{\left(C_{i_{1} j}(t) \geq \hat{C}_{i_{1} j}(t)\right)} \mid \hat{\tilde{\mathbf{q}}}(t)\right\}\right\} \\
& =g_{\mathrm{mdl}, j}(\hat{\tilde{\mathbf{q}}}(t)) \text {, }
\end{aligned}
$$

where inequality (a) results from the relation in (49). By taking the sum over all the channels, we can deduce that $g_{\text {mdl }}(\hat{\tilde{\mathbf{q}}}(t)) \geq$ $g_{\text {mpf }_{1}}(\hat{\tilde{\mathbf{q}}}(t))$.

- Step 2: We now want to show that system 'mdl' achieves a fraction $\left(1-\frac{1}{T_{\mathrm{b}}}\right) \frac{p_{\mathrm{c}}^{\min }}{\eta}$ of the stability region of system ' $\mathrm{pf}_{1}$ '.
Let us first define $D_{i}(t)$ as the service rate allocated for user $i$ at time-slot $t$. In addition, we define the quadratic Lyapunov function as the following

$$
L y(\mathbf{x}) \triangleq \frac{1}{2}(\mathbf{x} \cdot \mathbf{x})=\frac{1}{2} \sum_{i=1}^{N} x_{i}^{2} .
$$

Considering $T_{\mathrm{b}}$ slots into the future, the evolution equation for queue $q_{i}$, for all $i \in\{1, \ldots, N\}$, can be given as the following

$$
\begin{aligned}
& q_{i}\left((n+1) T_{\mathrm{b}}+d\right)=\max \left\{q_{i}\left(n T_{\mathrm{b}}+d\right)+\right. \\
& \left.\sum_{t_{1}=0}^{T_{\mathrm{b}}-1} A_{i}\left(n T_{\mathrm{b}}+d+t_{1}\right)-\sum_{t_{1}=1}^{T_{\mathrm{b}}-1} D_{i}\left(n T_{\mathrm{b}}+d+t_{1}\right), 0\right\},
\end{aligned}
$$

where the sum over the $D_{i}$ starts from 1 because every $T_{\mathrm{b}}$ slots the BS uses the first slot, i.e. slot $n T_{\mathrm{b}}$, to broadcast the queue lengths, i.e. no transmission occurs during this slot. For notational convenience we sometimes will replace $n T_{\mathrm{b}}+d$ by $t_{2}$, i.e. $t_{2}=n T_{\mathrm{b}}+d$. It is noteworthy to mention that considering $T_{\mathrm{b}}$ slots into the future is just for sake of simplifying the analysis, and that any sufficiently large positive integer can be considered to be the number of steps (i.e. slots) into the future. From (52) we have

$$
\begin{aligned}
& q_{i}^{2}\left(t_{2}+T_{\mathrm{b}}\right) \\
& \leq q_{i}^{2}\left(t_{2}\right)+\left[\sum_{t_{1}=0}^{T_{\mathrm{b}}-1} A_{i}\left(t_{2}+t_{1}\right)\right]^{2}+\left[\sum_{t_{1}=1}^{T_{\mathrm{b}}-1} D_{i}\left(t_{2}+t_{1}\right)\right]^{2} \\
& \quad+2 q_{i}\left(t_{2}\right)\left[\sum_{t_{1}=0}^{T_{\mathrm{b}}-1} A_{i}\left(t_{2}+t_{1}\right)-\sum_{t_{1}=1}^{T_{\mathrm{b}}-1} D_{i}\left(t_{2}+t_{1}\right)\right] \\
& \leq q_{i}^{2}\left(t_{2}\right)+T_{\mathrm{b}}^{2} A_{\max }^{2}+\left(T_{\mathrm{b}}-1\right)^{2} R_{K}^{2}+ \\
& 2 q_{i}\left(t_{2}\right)\left[\sum_{t_{1}=0}^{T_{\mathrm{b}}-1} A_{i}\left(t_{2}+t_{1}\right)-\sum_{t_{1}=1}^{T_{\mathrm{b}}-1} D_{i}\left(t_{2}+t_{1}\right)\right]
\end{aligned}
$$

where the first inequality results from the following fact: for any $q \geq 0, A \geq 0, D \geq 0$, we have

$$
(\max \{q+A-D, 0\})^{2} \leq q^{2}+A^{2}+D^{2}+2 q(A-D) .
$$

The second inequality holds since $A_{i}(t) \leq A_{\max }$ and $D_{i}(t) \leq$ $R_{K}, \forall t$; recall that $R_{K}$ stands for the highest rate. From the above, setting $E=\frac{1}{2} N T_{\mathrm{b}}^{2} A_{\max }^{2}+\frac{1}{2} N\left(T_{\mathrm{b}}-1\right)^{2} R_{K}^{2}$, it follows that

$$
\begin{aligned}
& \operatorname{Ly}\left(\mathbf{q}\left((n+1) T_{\mathrm{b}}+d\right)\right)-\operatorname{Ly}\left(\mathbf{q}\left(n T_{\mathrm{b}}+d\right)\right) \\
& =\frac{1}{2} \sum_{i=1}^{N}\left[q_{i}^{2}\left(t_{2}+T_{\mathrm{b}}\right)-q_{i}^{2}\left(t_{2}\right)\right] \\
& \leq E+q_{i}\left(t_{2}\right)\left[\sum_{t_{1}=0}^{T_{\mathrm{b}}-1} A_{i}\left(t_{2}+t_{1}\right)-\sum_{t_{1}=1}^{T_{\mathrm{b}}-1} D_{i}\left(t_{2}+t_{1}\right)\right] .
\end{aligned}
$$

Let us define $\operatorname{Dr}\left(\mathbf{q}\left(n T_{\mathrm{b}}\right)\right)$ as the conditional Lyapunov drift for time instance $n T_{\mathrm{b}}$ :

$$
\operatorname{Dr}\left(\mathbf{q}\left(n T_{\mathrm{b}}\right)\right) \triangleq \mathbb{E}\left\{L y\left(\mathbf{q}\left(t_{2}+T_{\mathrm{b}}\right)\right)-L y\left(\mathbf{q}\left(t_{2}\right)\right) \mid \mathbf{q}\left(n T_{\mathrm{b}}\right)\right\} .
$$


Using (54), we have that $\operatorname{Dr}\left(\mathbf{q}\left(n T_{\mathrm{b}}\right)\right)$ for a general scheduling policy satisfies

$$
\begin{aligned}
& \operatorname{Dr}\left(\mathbf{q}\left(n T_{\mathrm{b}}\right)\right) \\
& \leq E+\sum_{i=1}^{N} q_{i}\left(t_{2}\right) \sum_{t_{1}=0}^{T_{\mathrm{b}}-1} \mathbb{E}\left\{A_{i}\left(t_{2}+t_{1}\right) \mid \mathbf{q}\left(n T_{\mathrm{b}}\right)\right\} \\
& \quad-\sum_{i=1}^{N} q_{i}\left(t_{2}\right) \sum_{t_{1}=1}^{T_{\mathrm{b}}-1} \mathbb{E}\left\{D_{i}\left(t_{2}+t_{1}\right) \mid \mathbf{q}\left(n T_{\mathrm{b}}\right)\right\} \\
& =E+T_{\mathrm{b}} \sum_{i=1}^{N} q_{i}\left(t_{2}\right) a_{i} \\
& \quad-\sum_{i=1}^{N} q_{i}\left(t_{2}\right) \sum_{t_{1}=1}^{T_{\mathrm{b}}-1} \mathbb{E}\left\{D_{i}\left(t_{2}+t_{1}\right) \mid \mathbf{q}\left(n T_{\mathrm{b}}\right)\right\}
\end{aligned}
$$

where we have used the fact that arrivals are i.i.d. over time-slots and thus independent of current queue lengths, i.e. $\mathbb{E}\left\{A_{i}\left(t_{2}+t_{1}\right) \mid \mathbf{q}\left(n T_{\mathrm{b}}\right)\right\}=\mathbb{E}\left\{A_{i}\left(t_{2}+t_{1}\right)\right\}=a_{i}$. Note that the conditional expectation at the right-hand-side of (56) is w.r.t. the randomly observed channel states. Let $\Delta_{\text {mdl }}$ denote the scheduling policy under system 'mdl'. Also, we use $\Delta_{\mathrm{pf}}$ and $\Delta_{\mathrm{pf}_{1}}$ to denote the scheduling policies under 'pf' and ' $\mathrm{pf}_{1}$ ', respectively. For the drift under $\Delta_{\text {mdl }}$ we have

$$
\begin{aligned}
& D r^{\left(\Delta_{\text {mdl }}\right)}\left(\mathbf{q}\left(n T_{\mathrm{b}}\right)\right) \leq E+T_{\mathrm{b}} \sum_{i=1}^{N} q_{i}\left(t_{2}\right) a_{i}- \\
& \sum_{i=1}^{N} q_{i}\left(t_{2}\right) \sum_{t_{1}=1}^{T_{\mathrm{b}}-1} \mathbb{E}\left\{D_{i}^{\left(\Delta_{\text {mdl }}\right)}\left(t_{2}+t_{1}\right) \mid \mathbf{q}\left(n T_{\mathrm{b}}\right)\right\},
\end{aligned}
$$

where $D_{i}^{\left(\Delta_{\text {mdl }}\right)}(t)$ is the service rate allocated for user $i$ at timeslot $t$ under system 'mdl' and its expression can be given as

$$
D_{i}^{\left(\Delta_{\text {mdl }}\right)}(t)=\sum_{j=1}^{L} \hat{C}_{i j}(t) S_{i j}(t) \hat{Y}_{i j}(t) \mathbb{1}_{\left(C_{i j}(t) \geq \hat{C}_{i j}(t)\right)} .
$$

Based on the evolution equation of the queue lengths and the facts that $A_{i}(t) \leq A_{\max }$ and $D_{i}(t) \leq R_{K}$, it can be seen that

$$
-\left(s_{2}-s_{1}\right) R_{K} \leq q_{i}\left(s_{2}\right)-q_{i}\left(s_{1}\right) \leq\left(s_{2}-s_{1}\right) A_{\max } .
$$

Thus, the following holds $-d R_{K} \leq q_{i}\left(t_{2}\right)-q_{i}\left(n T_{\mathrm{b}}\right) \leq d A_{\max }$, or equivalently

$$
q_{i}\left(n T_{\mathrm{b}}\right)-d R_{K} \leq q_{i}\left(t_{2}\right) \leq q_{i}\left(n T_{\mathrm{b}}\right)+d A_{\max },
$$

where we recall that $t_{2}=n T_{\mathrm{b}}+d$. Plugging the above into (57), we get

$$
\begin{aligned}
& D r^{\left(\Delta_{\text {mdl }}\right)}\left(\mathbf{q}\left(n T_{\mathrm{b}}\right)\right) \\
\leq & E+T_{\mathrm{b}} \sum_{i=1}^{N}\left(q_{i}\left(n T_{\mathrm{b}}\right)+d A_{\max }\right) a_{i}- \\
& \sum_{i=1}^{N}\left(q_{i}\left(n T_{\mathrm{b}}\right)-d R_{K}\right) \sum_{t_{1}=1}^{T_{\mathrm{b}}-1} \mathbb{E}\left\{D_{i}^{\left(\Delta_{\mathrm{mdl}}\right)}\left(t_{2}+t_{1}\right) \mid \mathbf{q}\left(n T_{\mathrm{b}}\right)\right\} \\
\leq & E+T_{\mathrm{b}} N d A_{\max }^{2}+\left(T_{\mathrm{b}}-1\right) N d R_{K}^{2}+T_{\mathrm{b}} \sum_{i=1}^{N} q_{i}\left(n T_{\mathrm{b}}\right) a_{i}- \\
& \sum_{i=1}^{N} q_{i}\left(n T_{\mathrm{b}}\right) \sum_{t_{1}=1}^{T_{\mathrm{b}}-1} \mathbb{E}\left\{D_{i}^{\left(\Delta_{\mathrm{mdl}}\right)}\left(t_{2}+t_{1}\right) \mid \mathbf{q}\left(n T_{\mathrm{b}}\right)\right\}
\end{aligned}
$$

Recall that $g_{\text {mdl }}(\hat{\tilde{\mathbf{q}}}(t))=\sum_{i=1}^{N} \hat{\tilde{q}}_{i}(t) \mathbb{E}\left\{D_{i}^{\left(\Delta_{\text {mdl }}\right)}(t) \mid \hat{\tilde{\mathbf{q}}}(t)\right\}$. By setting $t=t_{2}+t_{1}$, we have $\hat{\tilde{\mathbf{q}}}(t)=\mathbf{q}\left(n T_{\mathrm{b}}\right), \forall t_{1}$ such that $1 \leq t_{1} \leq T_{\mathrm{b}}-1$. This can be explained as follows. For timeslot $t$, under 'mdl' (using FSA) the feedback decision at timeslot $t-d$ is done based on $\hat{\tilde{\mathbf{q}}}(t)$; we recall that $\hat{\tilde{\mathbf{q}}}(t)=\tilde{\mathbf{q}}(t-d)$, where $\tilde{\mathbf{q}}(t-d)=\mathbf{q}\left(n T_{\mathrm{b}}\right)$, for $t-d \in\left[n T_{\mathrm{b}},(n+1) T_{\mathrm{b}}[\right.$. For each channel, only one link will report its feedback to the BS at time-slot $t-d$, so evidently this link will be transmitting over this channel at time-slot $t$. Here $t=t_{2}+t_{1}=n T_{\mathrm{b}}+d+t_{1}$, thus at time-slot $t-d=n T_{\mathrm{b}}+t_{1}$ the feedback decision is based on $\hat{\tilde{\mathbf{q}}}(t)=\tilde{\mathbf{q}}(t-d)=\tilde{\mathbf{q}}\left(n T_{\mathrm{b}}+t_{1}\right)$. But $\tilde{\mathbf{q}}\left(n T_{\mathrm{b}}+t_{1}\right)=\mathbf{q}\left(n T_{\mathrm{b}}\right), \forall t_{1}$ such that $1 \leq t_{1} \leq T_{\mathrm{b}}-1$, since the BS broadcasts the queue lengths at time-slots $0, T_{\mathrm{b}}, \ldots, n T_{\mathrm{b}}, \ldots$ Hence, the following holds

$$
\begin{aligned}
g_{\mathrm{mdl}}(\hat{\tilde{\mathbf{q}}}(t)) & =g_{\mathrm{mdl}}\left(\mathbf{q}\left(n T_{\mathrm{b}}\right)\right) \\
& =\sum_{i=1}^{N} q_{i}\left(n T_{\mathrm{b}}\right) \mathbb{E}\left\{D_{i}^{\left(\Delta_{\text {mdl }}\right)}(t) \mid \mathbf{q}\left(n T_{\mathrm{b}}\right)\right\} .
\end{aligned}
$$

On the other hand, we have

$$
g_{\mathrm{pf}_{1}}(\hat{\tilde{\mathbf{q}}}(t))=\sum_{i=1}^{N} \hat{\tilde{q}}_{i}(t) \mathbb{E}\left\{D_{i}^{\left(\Delta_{\mathrm{pf}_{1}}\right)}(t) \mid \hat{\tilde{\mathbf{q}}}(t)\right\}
$$

where $D_{i}^{\left(\Delta_{\mathrm{pf}_{1}}\right)}(t)=\sum_{j=1}^{L} C_{i j}(t) S_{i j}(t)$.

In a similar manner to 'mdl', it can be shown that

$$
\begin{aligned}
g_{\mathrm{pf}_{1}}(\hat{\tilde{\mathbf{q}}}(t)) & =g_{\mathrm{pf}_{1}}\left(\mathbf{q}\left(n T_{\mathrm{b}}\right)\right) \\
& =\sum_{i=1}^{N} q_{i}\left(n T_{\mathrm{b}}\right) \mathbb{E}\left\{D_{i}^{\left(\Delta_{\mathrm{pf}_{1}}\right)}(t) \mid \mathbf{q}\left(n T_{\mathrm{b}}\right)\right\} .
\end{aligned}
$$

Using the relation $g_{\mathrm{mdl}}(\hat{\tilde{\mathbf{q}}}(t)) \geq \frac{p_{\mathrm{c}}^{\min }}{\eta} g_{\mathrm{pf}_{1}}(\hat{\tilde{\mathbf{q}}}(t))$, which was shown earlier, we get

$$
\begin{aligned}
& \sum_{i=1}^{N} q_{i}\left(n T_{\mathrm{b}}\right) \sum_{t_{1}=1}^{T_{\mathrm{b}}-1} \mathbb{E}\left\{D_{i}^{\left(\Delta_{\mathrm{mdl}}\right)}\left(t_{2}+t_{1}\right) \mid \mathbf{q}\left(n T_{\mathrm{b}}\right)\right\} \geq \\
& \frac{p_{\mathrm{c}}^{\min }}{\eta} \sum_{i=1}^{N} q_{i}\left(n T_{\mathrm{b}}\right) \sum_{t_{1}=1}^{T_{\mathrm{b}}-1} \mathbb{E}\left\{D_{i}^{\left(\Delta_{\mathrm{pf}_{1}}\right)}\left(t_{2}+t_{1}\right) \mid \mathbf{q}\left(n T_{\mathrm{b}}\right)\right\} .
\end{aligned}
$$

Plugging the above directly into (61) yields

$$
\begin{aligned}
& D r^{\left(\Delta_{\mathrm{mdl}}\right)}\left(\mathbf{q}\left(n T_{\mathrm{b}}\right)\right) \leq E_{1}+T_{\mathrm{b}} \sum_{i=1}^{N} q_{i}\left(n T_{\mathrm{b}}\right) a_{i}- \\
& \frac{p_{\mathrm{c}}^{\min }}{\eta} \sum_{i=1}^{N} q_{i}\left(n T_{\mathrm{b}}\right) \sum_{t_{1}=1}^{T_{\mathrm{b}}-1} \mathbb{E}\left\{D_{i}^{\left(\Delta_{\mathrm{pf}_{1}}\right)}\left(t_{2}+t_{1}\right) \mid \mathbf{q}\left(n T_{\mathrm{b}}\right)\right\},
\end{aligned}
$$

in which $E_{1}=E+T_{\mathrm{b}} N d A_{\max }^{2}+\left(T_{\mathrm{b}}-1\right) N d R_{K}^{2}$. Since, by definition, policy $\Delta_{\mathrm{pf}_{1}}$ maximizes the weighted sum $\sum_{i=1}^{N} q_{i}\left(n T_{\mathrm{b}}\right) D_{i}\left(t_{2}+t_{1}\right)$ over all alternative decisions, we can write

$$
\sum_{i=1}^{N} q_{i}\left(n T_{\mathrm{b}}\right) D_{i}^{\left(\Delta_{\left.\mathrm{pf}_{1}\right)}\right)}\left(t_{2}+t_{1}\right) \geq \sum_{i=1}^{N} q_{i}\left(n T_{\mathrm{b}}\right) D_{i}^{(\Delta)}\left(t_{2}+t_{1}\right)
$$

in which $\Delta$ represents any alternative (possibly randomized) scheduling decision that can stabilize system ' $\mathrm{pf}_{1}$ '. Taking a 
conditional expectation of the above inequality (given $\mathbf{q}\left(n T_{\mathrm{b}}\right)$ ) yields

$$
\begin{aligned}
& \sum_{i=1}^{N} q_{i}\left(n T_{\mathrm{b}}\right) \mathbb{E}\left\{D_{i}^{\left(\Delta_{\mathrm{pf}_{1}}\right)}\left(t_{2}+t_{1}\right) \mid \mathbf{q}\left(n T_{\mathrm{b}}\right)\right\} \geq \\
& \sum_{i=1}^{N} q_{i}\left(n T_{\mathrm{b}}\right) \mathbb{E}\left\{D_{i}^{(\Delta)}\left(t_{2}+t_{1}\right) \mid \mathbf{q}\left(n T_{\mathrm{b}}\right)\right\},
\end{aligned}
$$

where we recall that $t_{2}=n T_{\mathrm{b}}+d$. Plugging the above into (66) yields

$$
\begin{aligned}
& \operatorname{Dr}^{\left(\Delta_{\text {mdl }}\right)}\left(\mathbf{q}\left(n T_{\mathrm{b}}\right)\right) \\
\leq & E_{1}+T_{\mathrm{b}} \sum_{i=1}^{N} q_{i}\left(n T_{\mathrm{b}}\right) a_{i}- \\
& \frac{p_{\mathrm{c}}^{\min }}{\eta} \sum_{i=1}^{N} q_{i}\left(n T_{\mathrm{b}}\right) \sum_{t_{1}=1}^{T_{\mathrm{b}}-1} \mathbb{E}\left\{D_{i}^{(\Delta)}\left(t_{2}+t_{1}\right) \mid \mathbf{q}\left(n T_{\mathrm{b}}\right)\right\} \\
= & E_{1}+T_{\mathrm{b}} \sum_{i=1}^{N} q_{i}\left(n T_{\mathrm{b}}\right) a_{i}- \\
& \frac{p_{\mathrm{c}}^{\min }}{\eta} \sum_{i=1}^{N} q_{i}\left(n T_{\mathrm{b}}\right) \mathbb{E}\left\{\sum_{t_{1}=1}^{T_{\mathrm{b}}-1} D_{i}^{(\Delta)}\left(t_{2}+t_{1}\right) \mid \mathbf{q}\left(n T_{\mathrm{b}}\right)\right\} .
\end{aligned}
$$

We now consider a particular policy $\Delta$ that depends only on the channels states. It is worth recalling that each channel process is not i.i.d. in time. We point out that the process $D_{i}^{(\Delta)}(t)$, defined over the channel convergent (fading) process, is rate convergent [22]. Using the definition of rate convergence for $D_{i}^{(\Delta)}(t)$ with rate $r_{i}^{(\Delta)}$, we have (refer to [22] for more details)

$$
\frac{1}{T_{\mathrm{rc}}} \sum_{t=0}^{T_{\mathrm{rc}}-1} D_{i}^{(\Delta)}(t) \rightarrow r_{i}^{(\Delta)} \text {, with probability } 1 \text { as } T_{\mathrm{rc}} \rightarrow \infty,
$$

and for any $\epsilon_{i}>0$ there exists an interval $T_{\mathrm{rc}}$ such that, for all initial times $t_{0}$, and regardless of past history, the following holds

$$
\left|r_{i}^{(\Delta)}-\mathbb{E}\left\{\frac{1}{T_{\mathrm{rc}}} \sum_{t=t_{0}}^{t_{0}+T_{\mathrm{rc}}-1} D_{i}^{(\Delta)}(t)\right\}\right| \leq \epsilon_{i} .
$$

By choosing $T_{\mathrm{rc}}=T_{\mathrm{b}}-1$ and $t_{0}=1$, we can deduce that

$$
\mathbb{E}\left\{\frac{1}{T_{\mathrm{b}}-1} \sum_{t_{1}=1}^{T_{\mathrm{b}}-1} D_{i}^{(\Delta)}\left(t_{1}+t_{2}\right) \mid \mathbf{q}\left(n T_{\mathrm{b}}\right)\right\} \geq r_{i}^{(\Delta)}-\epsilon_{i} .
$$

Note that $T_{\mathrm{b}}$ needs to be sufficiently large in order to get the $\epsilon_{i}$ sufficiently small. Plugging the above into (69) yields

$$
\begin{aligned}
& D r^{\left(\Delta_{\mathrm{mdll}}\right)}\left(\mathbf{q}\left(n T_{\mathrm{b}}\right)\right) \\
& \leq E_{1}+T_{\mathrm{b}} \sum_{i=1}^{N} q_{i}\left(n T_{\mathrm{b}}\right) a_{i}- \\
& \quad \frac{p_{\mathrm{c}}^{\min }}{\eta}\left(T_{\mathrm{b}}-1\right) \sum_{i=1}^{N} q_{i}\left(n T_{\mathrm{b}}\right)\left[r_{i}^{(\Delta)}-\epsilon_{i}\right] \\
& \leq E_{1}-\left(T_{\mathrm{b}}-1\right) \frac{p_{\mathrm{c}}^{\min }}{\eta} \sum_{i=1}^{N} q_{i}\left(n T_{\mathrm{b}}\right)\left[r_{i}^{(\Delta)}-\beta^{-1} a_{i}-\epsilon\right],
\end{aligned}
$$

where $\beta=\left(1-\frac{1}{T_{\mathrm{b}}}\right) \frac{p_{\mathrm{c}}^{\min }}{\eta}$ and $\epsilon=\max _{i} \epsilon_{i}$.

Recall that $\Lambda_{\mathrm{pf}_{1}}$ represents the stability region achieved under system ' $\mathrm{pf}_{1}$ '. Let us suppose that the mean arrival rate vector a is interior to fraction $\beta$ of region $\Lambda_{\mathrm{pf}_{1}}$, meaning that there exists an $\epsilon_{\max }$, which clearly depends on $\mathbf{a}$, such that

$$
\left(a_{1}+\epsilon_{\max }, \ldots, a_{N}+\epsilon_{\max }\right) \in \beta \Lambda_{\mathrm{pf}_{1}},
$$

or equivalently

$$
\left(\beta^{-1} a_{1}+\beta^{-1} \epsilon_{\max }, \ldots, \beta^{-1} a_{N}+\beta^{-1} \epsilon_{\max }\right) \in \Lambda_{\mathrm{pf}_{1}} .
$$

Thus, the following holds

$$
r_{i}^{(\Delta)} \geq \beta^{-1} a_{i}+\beta^{-1} \epsilon_{\max }, \forall i \in\{1, \ldots, N\} .
$$

Plugging the above inequality into (73) yields

$$
\operatorname{Dr}^{\left(\Delta_{\mathrm{mdll}}\right)}\left(\mathbf{q}\left(n T_{\mathrm{b}}\right)\right) \leq E_{1}-T_{\mathrm{b}}\left(\epsilon_{\max }-\beta \epsilon\right) \sum_{i=1}^{N} q_{i}\left(n T_{\mathrm{b}}\right) .
$$

Let $\breve{\epsilon}_{\max }=T_{\mathrm{b}}\left(\epsilon_{\max }-\beta \epsilon\right)$. Taking an expectation of $D r\left(\Delta_{\text {mdl }}\right)$ over the randomness of the queue lengths and summing over $n \in\{0,1, \ldots, T-1\}$ for some integer $T>0$, we get

$$
\begin{aligned}
& \mathbb{E}\left\{L y\left(\mathbf{q}\left(T T_{\mathrm{b}}+d\right)\right)\right\}-\mathbb{E}\{L y(\mathbf{q}(d))\} \leq \\
& E_{1} T-\breve{\epsilon}_{\max } \sum_{n=0}^{T-1} \sum_{i=1}^{N} \mathbb{E}\left\{q_{i}\left(n T_{\mathrm{b}}\right)\right\} .
\end{aligned}
$$

Rearranging terms, dividing by $\breve{\epsilon}_{\max } T$, and using the fact that $L y\left(\mathbf{q}\left(T T_{\mathrm{b}}+d\right)\right) \geq 0$ yields

$$
\frac{1}{T} \sum_{n=0}^{T-1} \sum_{i=1}^{N} \mathbb{E}\left\{q_{i}\left(n T_{\mathrm{b}}\right)\right\} \leq \frac{E_{1}}{\breve{\epsilon}_{\max }}+\frac{\mathbb{E}\{L y(\mathbf{q}(d))\}}{\breve{\epsilon}_{\max } T} .
$$

Assuming that $\mathbb{E}\{L y(\mathbf{q}(d))\}<\infty$ and taking a limsup, we eventually obtain

$$
\limsup _{T \rightarrow \infty} \frac{1}{T} \sum_{n=0}^{T-1} \sum_{i=1}^{N} \mathbb{E}\left\{q_{i}\left(n T_{\mathrm{b}}\right)\right\} \leq \frac{E_{1}}{\breve{\epsilon}_{\max }} .
$$

Based on the above inequality and the definition of strong stability, we can claim that $\Delta_{\text {mdl }}$ stabilizes any mean arrival rate vector interior to fraction $\beta$ of the stability region achieved under policy $\Delta_{\mathrm{pf}_{1}}$, meaning that $\Delta_{\text {mdl }}$ stabilizes the system for any arrivals such that $\mathbf{a} \in \beta \Lambda_{\mathrm{pf}_{1}}$.

Based on Lemma 1, we know that 'pf ${ }_{1}$ ' and 'pf' have the same stability region. Hence, we can deduce that $\Delta_{\text {mdl }}$ stabilizes any arrival rate vector interior to fraction $\beta$ of the stability region achieved under $\Delta_{\mathrm{pf}}$. This completes the proof.

\section{REFERENCES}

[1] M. Deghel, M. Assaad, and M. Debbah, "Opportunistic feedback reporting and scheduling scheme for multichannel wireless networks," in 2016 IEEE Global Communications Conference (GLOBECOM), Dec 2016, pp. 1-7.

[2] M. B. Stefania Sesia, Issam Toufik, "LTE, the UMTS long term evolution: From theory to practice." Wiley, 2009.

[3] D. Gesbert and M. S. Alouini, "How much feedback is multi-user diversity really worth?" in 2004 IEEE International Conference on Communications (IEEE Cat. No.04CH37577), vol. 1, June 2004, pp. 234-238.

[4] T. Tang and R. W. Heath, "Opportunistic feedback for downlink multiuser diversity," IEEE Communications Letters, vol. 9, no. 10, pp. 948 950, Oct 2005. 
[5] P. Svedman, S. K. Wilson, L. J. Cimini, and B. Ottersten, "Opportunistic beamforming and scheduling for OFDMA systems," IEEE Transactions on Communications, vol. 55, no. 5, pp. 941-952, May 2007.

[6] V. Hassel, D. Gesbert, M.-S. Alouini, and G. E. Oien, "A thresholdbased channel state feedback algorithm for modern cellular systems," IEEE Transactions on Wireless Communications, vol. 6, no. 7, pp. 24222426, July 2007.

[7] J. Chen, R. A. Berry, and M. L. Honig, "Limited feedback schemes for downlink OFDMA based on sub-channel groups," IEEE Journal on Selected Areas in Communications, vol. 26, no. 8, pp. 1451-1461, October 2008

[8] S. N. Donthi and N. B. Mehta, "Joint performance analysis of channel quality indicator feedback schemes and frequency-domain scheduling for LTE," IEEE Transactions on Vehicular Technology, vol. 60, no. 7, pp. 3096-3109, Sept 2011.

[9] L. Tassiulas and A. Ephremides, "Stability properties of constrained queueing systems and scheduling policies for maximum throughput in multihop radio networks," IEEE Transactions on Automatic Control, vol. 37, no. 12, pp. 1936-1948, Dec 1992.

[10] Y. Cui, V. K. N. Lau, R. Wang, H. Huang, and S. Zhang, "A survey on delay-aware resource control for wireless systems - large deviation theory, stochastic lyapunov drift, and distributed stochastic learning," IEEE Transactions on Information Theory, vol. 58, no. 3, pp. 16771701, March 2012.

[11] M. Karaca, Y. Sarikaya, O. Ercetin, T. Alpcan, and H. Boche, "Efficient wireless scheduling with limited channel feedback and performance guarantees," in 2012 IEEE 23rd International Symposium on Personal, Indoor and Mobile Radio Communications - (PIMRC), Sept 2012, pp. $106-111$.

[12] _ "Joint opportunistic scheduling and selective channel feedback," IEEE Transactions on Wireless Communications, vol. 12, no. 6, pp. 3024-3034, June 2013.

[13] C. Joo, X. Lin, and N. B. Shroff, "Understanding the capacity region of the greedy maximal scheduling algorithm in multihop wireless networks," IEEE/ACM Transactions on Networking, vol. 17, no. 4, pp. 1132-1145, Aug 2009.

[14] E. Modiano, D. Shah, and G. Zussman, "Maximizing throughput in wireless networks via gossiping," SIGMETRICS Perform. Eval. Rev. vol. 34, no. 1, pp. 27-38, Jun. 2006.

[15] S. Sanghavi, L. Bui, and R. Srikant, "Distributed link scheduling with constant overhead," SIGMETRICS Perform. Eval. Rev., vol. 35, no. 1, pp. 313-324, Jun. 2007.

[16] A. Gopalan, C. Caramanis, and S. Shakkottai, "On wireless scheduling with partial channel-state information," IEEE Transactions on Information Theory, vol. 58, no. 1, pp. 403-420, Jan 2012.

[17] W. Ouyang, S. Murugesan, A. Eryilmaz, and N. B. Shroff, "Scheduling with rate adaptation under incomplete knowledge of channel/estimator statistics," in 2010 48th Annual Allerton Conference on Communication, Control, and Computing (Allerton), Sept 2010, pp. 670-677.

[18] M. Ouyang and L. Ying, "Approaching throughput optimality with limited feedback in multichannel wireless downlink networks," IEEE/ACM Transactions on Networking, vol. 21, no. 6, pp. 1827-1838, Dec 2013.

[19] S. Bodas, S. Shakkottai, L. Ying, and R. Srikant, "Low-complexity scheduling algorithms for multi-channel downlink wireless networks," in 2010 Proceedings IEEE INFOCOM, March 2010, pp. 1-9.

[20] L. Ying and S. Shakkottai, "On throughput optimality with delayed network-state information," IEEE Transactions on Information Theory, vol. 57, no. 8, pp. 5116-5132, Aug 2011.

[21] M. Johnston and E. Modiano, "A new look at wireless scheduling with delayed information," in 2015 IEEE International Symposium on Information Theory (ISIT), June 2015, pp. 1407-1411.

[22] M. J. Neely, "Dynamic power allocation and routing for satellite and wireless networks with time varying channels," Ph.D. dissertation, Massachusetts Institute of Technology, 2003.

[23] L. Georgiadis, M. J, and R. Tassiulas, "Resource allocation and crosslayer control in wireless networks," in Foundations and Trends in Networking, 2006, pp. 1-149.

[24] A. Destounis, M. Assaad, M. Debbah, and B. Sayadi, "Traffic-aware training and scheduling for MISO wireless downlink systems," IEEE Transactions on Information Theory, vol. 61, no. 5, pp. 2574-2599, May 2015.

[25] A. A. Reddy, S. Banerjee, A. Gopalan, S. Shakkottai, and L. Ying, "Wireless scheduling with heterogeneously delayed network-state information," in 2010 48th Annual Allerton Conference on Communication, Control, and Computing (Allerton), Sept 2010, pp. 1577-1584.

[26] S. Haykin, Adaptive Filter Theory (3rd Ed.). Upper Saddle River, NJ, USA: Prentice-Hall, Inc., 1996.
Matha Deghel completed a double degree in September 2013 where he obtained his Engineering diploma from Lebanese University and his Master's degree from Telecom ParisTech \& Paris 6, both in Digital Communication Systems. In May 2017, he completed his Ph.D. in Telecommunications Engineering, on which he worked under the guidance of Prof. Mohamad Assaad and Prof. Mérouane Debbah, at CentraleSupélec. His research interests include resource optimization and cross-layer design in wireless networks, stochastic network optimization, and mathematical models of communication networks.

Mohamad Assaad received the MSc and $\mathrm{PhD}$ degrees, both in telecommunications, from Telecom ParisTech, Paris, France, in 2002 and 2006, respectively. Since 2006, he has been with the Telecommunications Department at CentraleSupélec, where he is currently a professor and holds the TCL Chair on $5 \mathrm{G}$ systems. He has co-authored 1 book and more than 90 publications in journals and conference proceedings and serves regularly as TPC member for several top international conferences. He is an Editor for IEEE Wireless Communications Letters and has served as Co-Chair of IEEE ICC 2017 Wireless Communications Symposium. He has given in the past successful tutorials on 5G systems at various conferences including IEEE ISWCS 15 and IEEE WCNC 16 conferences. His research interests include mathematical modeling of communication networks, MIMO systems, resource management and cross-layer design in wireless networks. He is a Senior Member of the IEEE.

Mérouane Debbah entered the Ecole Normale Supérieure de Cachan (France) in 1996 where he received his M.Sc and Ph.D. degrees respectively. He worked for Motorola Labs (Saclay, France) from 1999-2002 and the Vienna Research Center for Telecommunications (Vienna, Austria) until 2003. From 2003 to 2007, he joined the Mobile Communications department of the Institut Eurecom (Sophia Antipolis, France) as an Assistant Professor. Since 2007, he is a Full Professor at CentraleSupelec (Gif-sur-Yvette, France). From 2007 to 2014, he was the director of the Alcatel-Lucent Chair on Flexible Radio. Since 2014, he is Vice-President of the Huawei France R\&D center and director of the Mathematical and Algorithmic Sciences Lab. His research interests lie in fundamental mathematics, algorithms, statistics, information \& communication sciences research. He is an Associate Editor in Chief of the journal Random Matrix: Theory and Applications and was an associate and senior area editor for IEEE Transactions on Signal Processing respectively in 2011-2013 and 2013-2014. Mérouane Debbah is a recipient of the ERC grant MORE (Advanced Mathematical Tools for Complex Network Engineering). $\mathrm{He}$ is a IEEE Fellow and a WWRF Fellow. He has managed 8 EU projects and more than 24 national and international projects. He has received more than 17 best paper awards, among which the 2015 IEEE Communications Society Leonard G. Abraham Prize, the 2015 IEEE Communications Society Fred W. Ellersick Prize, the 2016 IEEE Communications Society Best Tutorial paper award and the 2017 Eurasip Best Paper Award. He is the recipient of the Mario Boella award in 2005, the IEEE Glavieux Prize Award in 2011 and the Qualcomm Innovation Prize Award in 2012

Anthony Ephremides received the Ph.D. degree in electrical engineering from Princeton University, Princeton, NJ, USA, in 1971. He holds the Cynthia Kim Professorship of Information Technology with the Electrical and Computer Engineering Department, University of Maryland, College Park, MD, USA, where he is a Distinguished University Professor and has a joint appointment with the Institute for Systems Research, of which he was among the founding members in 1986 . He has been with the University of Maryland since 1971. He is the author of several hundred papers, conference presentations, and patents, and has received numerous awards, including the IEEE Third Millennium Medal, the 2000 Outstanding Systems Engineering Faculty Award from the Institute for Systems Research, the Kirwan Faculty Research and Scholarship Prize from the University of Maryland in 2001, and the 2006 Aaron Wyner Award for Exceptional Service and Leadership to the IEEE Information Theory Society. His research interests lie in the areas of communication systems and networks and all related disciplines, such as information theory, control and optimization, wireless networks, and energy efficient systems. 